\title{
Diffusion quantum Monte Carlo and GW study of the electronic properties of monolayer and bulk hexagonal boron nitride
}

\author{
R. J. Hunt, B. Monserrat, V. Zólyomi and N. D. Drummond
}

\section{Published version information}

Citation: RJ Hunt et al. 'Diffusion quantum Monte Carlo and GW study of the electronic properties of monolayer and bulk hexagonal boron nitride.' Physical Review B, vol. 101, no. 20 (2020): 205115.

DOI: $\underline{10.1103 / P h y s R e v B .101 .205115}$

This version is made available in accordance with publisher policies. Please cite only the published version using the reference above. This is the citation assigned by the publisher at the time of issuing the APV. Please check the publisher's website for any updates. 


\title{
Diffusion quantum Monte Carlo and $G W$ study of the electronic properties of monolayer and bulk hexagonal boron nitride
}

\author{
R. J. Hunt, ${ }^{1}$ B. Monserrat, ${ }^{2}$ V. Zólyomi, ${ }^{3}$ and N. D. Drummond ${ }^{1}$ \\ ${ }^{1}$ Department of Physics, Lancaster University, Lancaster LA1 4YB, United Kingdom \\ ${ }^{2}$ Cavendish Laboratory, University of Cambridge, J. J. Thomson Avenue, Cambridge CB3 OHE, United Kingdom \\ ${ }^{3}$ Hartree Centre, STFC Daresbury Laboratory, Daresbury WA4 4AD, United Kingdom
}

(Received 2 March 2020; accepted 23 April 2020; published 11 May 2020)

\begin{abstract}
We report diffusion quantum Monte Carlo (DMC) and many-body $G W$ calculations of the electronic band gaps of monolayer and bulk hexagonal boron nitride $(\mathrm{hBN})$. We find the monolayer band gap to be indirect. $G W$ predicts much smaller quasiparticle gaps at both the single-shot $G_{0} W_{0}$ and the partially self-consistent $G W_{0}$ levels. In contrast, solving the Bethe-Salpeter equation on top of the $G W_{0}$ calculation yields an exciton binding energy for the direct exciton at the $K$ point in close agreement with the DMC value. Vibrational renormalization of the electronic band gap is found to be significant in both the monolayer and the bulk. Taking vibrational effects into account, DMC overestimates the band gap of bulk hBN, while $G W$ theory underestimates it.
\end{abstract}

DOI: 10.1103/PhysRevB.101.205115

\section{INTRODUCTION}

Two-dimensional (2D) materials have provided an exciting new frontier for experimental and theoretical nanoscience in the 15 years since the first isolation of atomically thin layers of graphene by mechanical exfoliation from graphite $[1,2]$. In addition to graphene and its derivatives, the last few years have witnessed growing interest in semiconducting 2D materials such as transition-metal dichalcogenides [3-5] and phosphorene [6-8]. A recent trend has been the study of stacked heterostructures of 2D materials [9]. Heterostructures involving graphene and hexagonal boron nitride (hBN) have received particular attention because monolayer $\mathrm{hBN}$ is an insulating, atomically thin 2D material with a similar lattice constant to graphene and is therefore the ideal substrate for graphene-based electronics [10-14]. Monolayer or few-layer $\mathrm{hBN}$ is potentially an important component in novel electronic devices based on 2D materials, such as vertical tunneling diodes [15,16] and supercapacitors [17]. In addition, due to the slight lattice mismatch, graphene placed on hBN exhibits a moiré pattern with a period of up to $14 \mathrm{~nm}$ [18], and the resulting superlattices allow the experimental observation of exotic phenomena such as the formation of Hofstadter's butterfly [19] features in the band structure in the presence of a magnetic field [20,21]. Despite the importance of 2D hBN in current or proposed graphene-based electronics research, the properties of monolayers of hBN are not currently well characterized due to the experimental challenge of isolating and studying monolayers. In this paper, we use advanced theoretical electronic-structure methods to provide basic information about the size and nature of the electronic band gap of monolayers of hBN. We find that the gap of hBN monolayers is in principle indirect (so that optical transitions involve the absorption or emission of phonons), and that the quasiparticle gap is considerably enhanced relative to the bulk. However, the conduction band around its minimum at the
$\Gamma$ point is a free-electron-gas-like state that is only weakly bound to the hBN monolayer and has a relatively small spatial overlap with the valence states [22]; hence, the dipole matrix element for an optical transition from the valence-band maximum to the conduction-band minimum is inevitably small. Furthermore, the precise energy of a state that extends outside the layer will be strongly affected by the environment in which the layer finds itself (substrate, encapsulation, etc.). Hence, we expect inverse photoemission measurements to show the energy of the conduction band at $\Gamma$ to depend strongly on the environment. Likewise, the effective height of the energy barrier presented by an hBN monolayer in a vertical-tunneling experiment will depend sensitively on the environment of the layer.

Bulk hBN (also known as white graphite) consists of layers of boron and nitrogen atoms occupying the $A$ and $B$ hexagonal sublattice sites of a 2D honeycomb lattice. These layers are weakly bound together by van der Waals interactions, resulting in both the lubricating properties of $\mathrm{hBN}$ and the possibility of isolating monolayers by mechanical exfoliation. Bulk hBN adopts an $A A^{\prime}$ stacking arrangement in which each boron atom (with a positive partial charge) has a nitrogen atom (with a negative partial charge) vertically above it and vice versa. Whereas pristine graphene is a gapless semiconductor, monolayer hBN is an insulator due to the lack of sublattice symmetry. Bulk hBN is semiconducting, with experimental estimates of the band gap ranging from 5.2(2)7.1(1) eV [23-27]. Watanabe et al. find the quasiparticle band gap to be direct and of value $5.971 \mathrm{eV}$ in a single-crystal sample [25]. More recent experimental work by Cassabois et al. has indicated that bulk hBN is in fact an indirect semiconductor with a quasiparticle band gap of $6.08 \mathrm{eV}$ [27]. The experimental work of Cassabois et al., together with subsequent theoretical works [28,29], have elucidated the role of vibrational effects in phonon-assisted indirect optical transitions in bulk hBN. Many-body $G W$ calculations also 
indicate that bulk hBN is an indirect-gap semiconductor, with a fundamental gap of 5.95-6.04 eV between the valence-band maximum (which is near the $K$ point, on the $\Gamma \rightarrow K$ line) and the conduction-band minimum at $M$ [30-32].

One of the many reasons for the high levels of interest in $2 \mathrm{D}$ materials is that the electronic properties of monolayers often differ significantly from those of the bulk-layered material. Density functional theory (DFT) within the local density approximation (LDA) predicts the indirect band gap of monolayer hBN to be $4.6 \mathrm{eV}$ [22], and the $G W_{0}$ shift in the quasiparticle band gap is about $3.6 \mathrm{eV}$ [32], giving a gap of $8.2 \mathrm{eV}$ for the monolayer. Clearly the gap is considerably enhanced on going from bulk $\mathrm{hBN}$ to a monolayer. Bulk $\mathrm{hBN}$ is believed to exhibit a large exciton binding energy, with values of $0.7-1.2 \mathrm{eV}$ [31-33] predicted by $G W$-BetheSalpeter-equation ( $G W$-BSE) calculations. On the other hand, experimental measurements find the exciton binding energy to be only $0.13-0.15 \mathrm{eV}[25,27]$, although there are questions over the interpretation of these experimental results [29]. Exciton binding is further enhanced in a free-standing monolayer due to the reduction in screening. Indeed, $G W$-BSE calculations find that the exciton binding energy increases to $2.1 \mathrm{eV}$ in the monolayer [32].

Isolating monolayer $\mathrm{hBN}$ by exfoliation from bulk $\mathrm{hBN}$ has proved challenging, although Elias et al. have recently succeeded in growing atomically thin samples of hBN on graphite substrates [34]. Their reflectance and photoluminescence measurements indicate a direct gap of $6.1 \mathrm{eV}$ for $\mathrm{hBN}$ on graphite. However, the electronic properties of an isolated hBN monolayer (i.e., a freely suspended sample) are at present only accessible through theoretical calculations. Unfortunately, DFT systematically underestimates electronic band gaps and even many-body $G W$ methods [35] suffer from limitations, as exemplified by the disagreement between the self-consistent and non-self-consistent variants of the method when applied to hBN [32]. We have therefore made use of quantum Monte Carlo (QMC) methods [36,37] to study manybody effects in the band gap. We have calculated the electronic band gaps for excitations from the valence band at the $K$ point of the hexagonal Brillouin zone $\left(K_{\mathrm{v}}\right)$ to the conduction band at the $\Gamma$ and $K$ points $\left(\Gamma_{\mathrm{c}}\right.$ and $K_{\mathrm{c}}$ ) of monolayer hBN. In our DFT and $G W$ calculations, and our QMC calculations for bulk $\mathrm{hBN}$, we have also considered the conduction band at the $M$ point $\left(M_{\mathrm{c}}\right)$. Furthermore, we have investigated the effects of the vibrational renormalization of the electronic structure at the DFT level.

We have made use of two QMC methods: variational Monte Carlo (VMC) and diffusion Monte Carlo (DMC) [37]. In VMC, Monte Carlo integration is used to evaluate quantum mechanical expectation values with respect to trial wavefunction forms of arbitrary complexity. Free parameters in the trial wave functions are optimized by a variational approach. DMC involves simulating drifting, diffusion, and birth/death processes governed by the Schrödinger equation in imaginary time to project out the ground-state component of a trial wave function [36]. The fixed-node approximation [38] is used to maintain fermionic antisymmetry. All our QMC calculations were performed using the CASINO code [39].

QMC methods have only recently been applied to calculate the energy gaps of 2D materials [40,41]. A major challenge is the need to extrapolate the QMC band gaps to the thermodynamic limit of large system size because the computational expense of the method necessitates the use of relatively small simulation supercells subject to periodic boundary conditions [40]. In this paper we investigate finite-size effects in the band gap of hBN.

The rest of this paper is arranged as follows. In Sec. II we describe our DFT, $G W$, and QMC methodologies. We present our results in Sec. III. Finally, we draw our conclusions in Sec. IV. We use Hartree atomic units (a.u.) throughout, in which $\hbar=m_{\mathrm{e}}=|e|=4 \pi \epsilon_{0}=1$, except where other units are given explicitly.

\section{COMPUTATIONAL METHODOLOGY}

\section{A. DFT}

\section{Geometry optimization, lattice dynamics, and band-structure calculations}

We performed our DFT calculations using the LDA, the Perdew-Burke-Ernzerhof (PBE) generalized-gradientapproximation exchange-correlation functional [42], and the Heyd-Scuseria-Ernzerhof (HSE06) hybrid functional [43,44]. We used the CASTEP [45] and VASP [46] plane-wave-basis DFT codes. Our DFT-LDA and DFT-PBE relaxations of the lattice parameter used an artificial periodicity of $21.17 \AA$ in the out-of-plane direction, a $53 \times 53$ Monkhorst-Pack k-point grid, ultrasoft pseudopotentials, and a plane-wave cutoff of $680 \mathrm{eV}$. The same parameters were used in our calculations of the electronic band structure. Our phonon calculations used density functional perturbation theory [47], norm-conserving DFT pseudopotentials, a plane-wave cutoff energy of $1361 \mathrm{eV}$, an artificial periodicity of $26.46 \AA$, and a $53 \times 53$ MonkhorstPack k-point grid for both the electronic orbitals and the vibrational normal modes. In our DFT-HSE06 calculations of the lattice parameter and band structure we used an artificial periodicity of $15.875 \AA$ in the out-of-plane direction, an $11 \times 11$ Monkhorst-Pack k-point grid, norm-conserving DFT pseudopotentials, and a plane-wave cutoff of $816 \mathrm{eV}$.

\section{QMC trial-wave-function generation}

The DFT calculations performed to generate trial wave functions for our QMC calculations used Dirac-Fock pseudopotentials [48,49], a plane-wave cutoff energy of $2721 \mathrm{eV}$, and, in the monolayer case, an artificial periodicity of $18.52 \AA$ (apart from the $3 \times 3$ supercell, where the plane-wave cutoff energy and artificial periodicity were $2177 \mathrm{eV}$ and $13.35 \AA$, respectively).

We found that replacing PBE ultrasoft pseudopotentials with Trail-Needs Dirac-Fock pseudopotentials changes the monolayer $K_{\mathrm{v}} \rightarrow \Gamma_{\mathrm{c}}$ and $K_{\mathrm{v}} \rightarrow K_{\mathrm{c}}$ DFT-PBE gaps from 4.69 to $4.71 \mathrm{eV}$ and from 4.67 to $4.79 \mathrm{eV}$, respectively. In these calculations, the lattice parameter is fixed at the DFT-PBE value $a=2.512 \AA$, obtained with the PBE ultrasoft pseudopotentials. This suggests that the choice of pseudopotential introduces an uncertainty of around $0.1 \mathrm{eV}$ into our QMC gap estimates. 


\section{B. $G W(-B S E)$ calculations}

In the $G W$ approximation, many-body interactions are taken into account in a quasiparticle picture in which the screened Coulomb interaction $W$ between particles is included in the self-energy to first order. Varying levels of approximation are possible: the so-called single-shot $G_{0} W_{0}$ approach calculates the Green's function $G$ and the dielectric screening in the Coulomb interaction $W$ from DFT wave functions, while the partially and fully self-consistent $G W_{0}$ and $G W$ methods iterate one or both of these quantities until self-consistency is achieved. Excitonic effects in the optical absorption can be taken into account by solving the Bethe-Salpeter equation (BSE) following the $G W$ calculations. We performed $G_{0} W_{0}(-\mathrm{BSE})$ and $G W_{0}(-\mathrm{BSE})$ calculations for monolayer $\mathrm{hBN}$, and for test purposes also $G_{0} W_{0}$ and $G W_{0}$ calculations for bulk hBN.

In our $G W$ calculations we used the VASP [46] plane-wavebasis code for bulk hBN. The HSE06 functional $[43,44]$ was used to calculate the orbitals and their derivatives as input for the single-shot $G_{0} W_{0}$ calculations [50]. Convergence of the $G_{0} W_{0}$ calculation with respect to its principal convergence parameters was achieved using a $12 \times 12 \times 12$ MonkhorstPack k-point grid, with 24 electronic bands taken into account, and a plane-wave cutoff energy of $400 \mathrm{eV}$. These parameters converge the band gap of bulk hBN to within $0.1 \mathrm{eV}$. We used the same parameter set to compute the partially selfconsistent $G W_{0}$ band gap. The results of the bulk calculations are discussed in Sec. III C.

For the monolayer $G W$ calculations we used the BERKELEYGW code [51] in order to be able to treat a much larger number of empty bands. In the bulk, the $G_{0} W_{0}$ gap changes by less than $50 \mathrm{meV}$ when the number of electronic bands is increased from 24 to 48 . In contrast, the monolayer requires 1200 bands to be taken into account for the same level of convergence; otherwise, the dielectric function is too inaccurate to predict reliable self-energy corrections. The k-point grid for the monolayer calculations was set to $24 \times 24 \times 1$, while the plane-wave cutoff during the many-body calculations was set to $408.17 \mathrm{eV}$ (30 Ry). In these calculations, the DFT wave functions were calculated using the PBE functional. For the monolayer, the optical absorption coefficient was also calculated at both the single-shot and the $G W_{0}$ level by solving the Bethe-Salpeter equation. In both cases we took six empty and four occupied bands into account. Truncation of the Coulomb interaction was applied in the monolayer calculations.

\section{QMC calculations}

\section{Evaluating quasiparticle and excitonic gaps}

To calculate an excitation energy using DMC we exploit the fixed-node approximation [38] and evaluate the difference of the total energies obtained using trial wave functions corresponding to the ground state and the particular excited state of interest [52-54]. For each excited state an appropriate wave function can be constructed by choosing the occupancies of the orbitals in the Slater determinants (see Sec. II C 2). The DMC energy of an excited state is exact if the nodal surface of the trial wave function is exact, as is the case for the ground state, although the DMC energy is only guaranteed to be an upper bound on the energy for certain excited states [55].

The quasiparticle bands at a particular point may be evaluated as $\mathcal{E}_{i}(\mathbf{k})=E^{+}(\mathbf{k}, i)-E^{\mathrm{GS}}$ for unoccupied states, where $E^{+}(\mathbf{k}, i)$ is the total energy when an electron is added to the system and occupies band $i$ at wave vector $\mathbf{k}$ and $E^{\mathrm{GS}}$ is the ground-state total energy. For occupied states we evaluate $\mathcal{E}_{i}(\mathbf{k})=E^{\mathrm{GS}}-E^{-}(\mathbf{k}, i)$, where $E^{-}(\mathbf{k}, i)$ is the total energy when an electron is removed from band $i$ at wave vector k. The quasiparticle band gap $\Delta_{\mathrm{qp}}$ is the difference of the energy bands at the conduction-band minimum (CBM) and valence-band maximum (VBM):

$$
\Delta_{\mathrm{qp}}=\mathcal{E}_{\mathrm{CBM}}-\mathcal{E}_{\mathrm{VBM}}=E_{\mathrm{CBM}}^{+}+E_{\mathrm{VBM}}^{-}-2 E^{\mathrm{GS}} .
$$

The excitonic gap $\Delta_{\mathrm{ex}}$ is defined as the difference in energy when an electron is promoted from the VBM to the CBM:

$$
\Delta_{\mathrm{ex}}=E_{\mathrm{VBM} \rightarrow \mathrm{CBM}}^{\mathrm{pr}}-E^{\mathrm{GS}},
$$

where $E_{\mathrm{VBM} \rightarrow \mathrm{CBM}}^{\mathrm{pr}}$ is the total energy evaluated with a trial wave function in which an electron has been promoted from the VBM to the CBM. In Sec. II C 5 we investigate whether it is important to construct appropriate wave functions for excitonic spin singlets or triplets when calculating gaps.

\section{Trial wave functions}

We used Slater-Jastrow (SJ) trial wave functions $\Psi=$ $\exp (J) S^{\uparrow} S^{\downarrow}$ in our QMC calculations. The Slater determinants for up- and down-spin electrons $S^{\uparrow}$ and $S^{\downarrow}$ contained Kohn-Sham orbitals generated using the CASTEP plane-wavebasis code [45] in a three-dimensionally periodic cell, as described in Sec. II A 2. However, the orbitals were rerepresented in a localized "blip" B-spline basis [56] to improve the scaling of the QMC calculations with system size and to allow us, in the monolayer case, to discard the artificial periodicity in the out-of-plane direction. The Jastrow factor $\exp (J)$ is a positive, symmetric, explicit function of interparticle distances. We used the Jastrow form described in Ref. [57], in which the Jastrow exponent $J$ consists of short-range, isotropic electron-electron, electron-nucleus, and electron-electron-nucleus terms, which are polynomials in the interparticle distances, as well as long-range electron-electron terms expanded in plane waves. The free parameters in our Jastrow factors were optimized by unreweighted variance minimization [58,59].

Within Hartree-Fock theory, band gaps are significantly overestimated due to the tendency to overlocalize electronic states in a theory that does not allow correlation to keep electrons apart. DMC retrieves a large but finite fraction of the correlation energy. Assuming the fraction of correlation energy retrieved in the ground state is similar to or greater than the fraction retrieved in an excited state, we expect the DMC gaps to be upper bounds on the true gaps. If we increase the fraction of correlation energy retrieved, e.g., by including a backflow transformation $[60,61]$, then (if anything) we expect to see a decrease in the band gap.

We performed some test calculations with Slater-Jastrowbackflow (SJB) trial wave functions [60,61]. In a backflow wave function the orbitals in the Slater determinant are evaluated not at the actual electron positions, but at quasiparticle 
TABLE I. DMC ground-state (GS) energy per primitive cell, quasiparticle band gap, and excitonic band gap of monolayer hBN in a supercell consisting of $3 \times 3$ primitive cells, as obtained using different trial wave functions and time steps. The $\mathbf{k}$-vector grid includes both $\Gamma$ and $K$. Where the time step is 0 , the reported results have been obtained by linear extrapolation to zero time step. The fact that the excitonic gap is higher than the quasiparticle gap is a manifestation of finite-size error in the uncorrected gaps, as explained in Sec. II C 7.

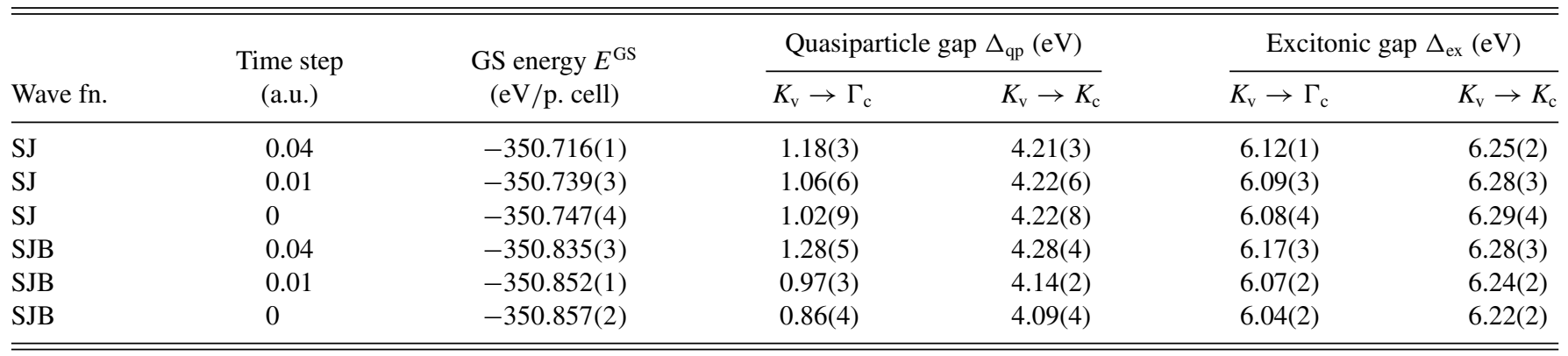

positions that are functions of all the particle coordinates. The backflow function, which describes the offset of the quasiparticle coordinates relative to the actual coordinates, contains free parameters to be determined by an optimization method. The Jastrow factor and backflow functions were optimized by VMC energy minimization [62]. As shown in Table I, backflow lowers the DMC total energies significantly. However, the amount by which backflow reduces the quasiparticle and excitonic gaps is small: about 0.10(3) $\mathrm{eV}$ on average. We investigated the reoptimization of backflow functions in the supercells in which an electron has been added or removed, finding that reoptimization raises the gap slightly. This is perhaps indicative of static correlation (multireference character) effects in the nodal surface that are not addressed by the use of backflow. Since QMC simulations with backflow are significantly more expensive, and finite-size effects are a potentially dominant source of error in our work, we did not use backflow in our production calculations.

Apart from these tests we have used the ground-stateoptimized Jastrow factor (and backflow function, where applicable) in all our excited-state calculations. The fixed-node SJ-DMC energy does not depend on the Jastrow factor, except via the pseudopotential locality approximation [63], and so reoptimizing the Jastrow factor in each excited state would be pointless in any case. The single-particle bands at $K$ and $K^{\prime}$ are degenerate, and hence we can construct multideterminant excited-state wave functions from the degenerate orbitals. We discuss this in Sec. II C 6.

\section{DMC time step, etc.}

The time-step error in the total energy per primitive cell is clearly significant, as shown in Table I; however, there is a partial cancellation of time-step errors when we take differences of total energies to obtain gaps. For the SJ-DMC gaps the time-step errors are of marginal significance. Nevertheless, since we would like to achieve very high accuracy, we have used DMC time steps of 0.01 and 0.04 a.u. and extrapolated our results linearly to zero time step. The timestep errors in our SJB-DMC gaps are considerably larger. All our DMC calculations used populations of at least 1024 walkers, making population-control bias negligible. We used Dirac-Fock pseudopotentials [48,49] to represent the boron and nitrogen atoms, including core-polarization corrections [64].

\section{Comparison of VMC and DMC gap results}

VMC is considerably cheaper than DMC, typically by a factor of at least 10. VMC can therefore be used to study larger systems than DMC. However, whereas fixed-node DMC total energies and band gaps are independent of the Jastrow factor in the limit of zero time step and large population, VMC energies are determined by the Jastrow factor. The use of a stochastically optimized Jastrow factor is therefore an additional source of noise in the VMC gaps. VMC and DMC results obtained with different levels of trial wave function for a $3 \times 3$ supercell are presented in Table II. The trial wave function in the "Hartree-Fock" VMC calculations was simply a Slater determinant of DFT-PBE orbitals, with no description of correlation. The fractions of correlation energy retrieved at the SJ-VMC and SJB-VMC levels are clearly different in the ground and excited states. However, we find the VMC gaps to be larger than the DMC gaps, and the SJ gaps to be larger than the SJB ones, as expected. We do not believe our VMC results can be used to aid the extrapolation of our DMC gaps to the thermodynamic limit of infinite system size.

\section{Singlet and triplet excitations}

We have calculated the SJ-DMC energy difference between the singlet and triplet excitonic states in a $3 \times 3$ supercell of hBN. We used single-determinant trial wave functions in which an electron was promoted with and without a spin flip to describe the triplet and singlet states, respectively [65]. The orbitals were generated in a non-spin-polarized groundstate DFT-PBE calculation. The singlet excitonic state for a promotion from $K_{\mathrm{V}} \rightarrow K_{\mathrm{c}}$ is $0.12(2) \mathrm{eV}$ lower in energy than the triplet state. For a promotion from $K_{\mathrm{v}} \rightarrow \Gamma_{\mathrm{c}}$, the energy difference between the singlet and triplet excitonic states is statistically insignificant (smaller than the error bar of $0.02 \mathrm{eV}$ ). Because these results were obtained in a small $(3 \times 3)$ supercell, these estimates of the singlet-triplet splitting should only be regarded as being of qualitative accuracy.

In summary, the energy difference between the singlet and triplet excitonic states in hBN appears to be small, especially when the electron and hole have different wave vectors. Apart 
TABLE II. "Hartree-Fock" VMC (HFVMC), SJ-VMC, SJB-VMC, SJ-DMC, and SJB-DMC ground-state (GS) total energies, energy variances, quasiparticle $(\mathrm{QP})$ gaps, and excitonic gaps for a $3 \times 3$ supercell of monolayer hBN. The fact that the excitonic gap is higher than the quasiparticle gap is a manifestation of finite-size error in the uncorrected gaps, as explained in Sec. IIC 7.

\begin{tabular}{|c|c|c|c|c|c|c|}
\hline \multirow[b]{2}{*}{ Method } & \multirow{2}{*}{$\begin{array}{c}\text { GS energy } E^{\mathrm{GS}} \\
(\mathrm{eV} / \text { p. cell })\end{array}$} & \multirow{2}{*}{$\begin{array}{l}\text { Var. } \sigma^{2} \\
\text { (a.u.) }\end{array}$} & \multicolumn{2}{|c|}{$\mathrm{QP}$ gap $\Delta_{\mathrm{qp}}(\mathrm{eV})$} & \multicolumn{2}{|c|}{ Exc. gap $\Delta_{\mathrm{ex}}(\mathrm{eV})$} \\
\hline & & & $K_{\mathrm{v}} \rightarrow \Gamma_{\mathrm{c}}$ & $K_{\mathrm{v}} \rightarrow K_{\mathrm{c}}$ & $K_{\mathrm{v}} \rightarrow \Gamma_{\mathrm{c}}$ & $K_{\mathrm{v}} \rightarrow K_{\mathrm{c}}$ \\
\hline HFVMC & $-341.961(4)$ & 21.39 & $2.63(8)$ & $5.95(8)$ & $7.13(5)$ & $7.65(5)$ \\
\hline SJ-VMC & $-349.8780(4)$ & 3.18 & $2.559(9)$ & $4.593(9)$ & $7.118(6)$ & $6.378(5)$ \\
\hline SJB-VMC & $-350.229(2)$ & 2.11 & $2.55(4)$ & $4.46(4)$ & $7.18(2)$ & $6.30(2)$ \\
\hline SJ-DMC & $-350.747(4)$ & & $1.02(9)$ & $4.22(8)$ & $6.08(4)$ & $6.29(4)$ \\
\hline SJB-DMC & $-350.857(2)$ & & $0.86(4)$ & $4.09(4)$ & $6.04(2)$ & $6.22(2)$ \\
\hline
\end{tabular}

from these tests, all the exited-state calculations reported in this paper used singlet excitations.

\section{Multideterminant wave functions}

We have considered three different ways of describing the wave function of a singlet excitonic state: (i) simply promoting a single electron from one state to another without changing its spin in a single-determinant wave function; (ii) constructing a two-determinant wave function in which spinup and -down electrons are promoted in the first and second determinants, respectively; and (iii) constructing a multideterminant wave function consisting of a linear combination of all the degenerate excited-state determinants (i.e., accounting for the degeneracy of $K$ and $K^{\prime}$ as well as spin degeneracy). In case (iii), we have four- and eight-determinant wave functions for excitations from $K_{\mathrm{v}} \rightarrow \Gamma_{\mathrm{c}}$ and from $K_{\mathrm{v}} \rightarrow K_{\mathrm{c}}$, respectively. We optimized the determinant expansion coefficients for these multideterminant wave functions in the presence of a fixed Jastrow factor that was optimized in the ground state, but we did not find a statistically significant reduction in the VMC energy. Any reduction in the DMC energy would be even smaller and hence we conclude that, to the level of precision at which we are working, there is no advantage to using such a multideterminant wave function. This does not imply that larger multideterminant wave functions would not significantly reduce fixed-node errors.

\section{Finite-size effects}

We have performed QMC calculations for monolayer $\mathrm{hBN}$ in a range of hexagonal supercells, from $2 \times 2$ to $9 \times 9$ primitive cells. Choosing the monolayer supercell to be hexagonal maximizes the distance between nearest periodic images of particles, and is therefore expected to minimize finitesize effects. For bulk hBN the choice of supercell is more complicated. In general, a supercell is defined by an integer "supercell matrix" $S$ such that

$$
\mathbf{a}_{i}^{\mathrm{S}}=\sum_{k} S_{i k} \mathbf{a}_{k}^{\mathrm{P}},
$$

where $\mathbf{a}_{k}^{\mathrm{P}}$ is the $k$ th primitive lattice vector and $\mathbf{a}_{i}^{\mathrm{S}}$ is the $i$ th supercell lattice vector. The supercell defined by $S$ contains $N_{\mathrm{P}}=|\operatorname{det}(S)|$ primitive cells. For a given number of primitive cells $N_{\mathrm{P}}$ we may therefore search over integer supercell matrices $S$ such that $|\operatorname{det}(S)|=N_{\mathrm{P}}$ to find the supercell matrix for which the nearest-image distance is maximized [66]. In general, the optimal supercell matrix is nondiagonal. Our bulk
hBN supercells contained $N_{\mathrm{P}}=9,18,27$, and 36 primitive cells. Unlike the monolayer, in bulk $\mathrm{hBN}$ we are unable to choose a large set of geometrically similar supercells that both maximize the distance between periodic images and have a tractable number of particles.

Different choices of supercell Bloch vector $[67,68]$ allow one to obtain different points on the electronic band structure in a finite supercell [52-54]. In the monolayer, if one uses a $3 m \times 3 n$ supercell, where $m$ and $n$ are natural numbers, with the supercell Bloch vector being $\mathbf{k}_{\mathrm{s}}=\mathbf{0}$, then the set of orbitals in the trial wave function includes the bands at both the $\Gamma$ and the $K$ points of the primitive-cell Brillouin zone. In this case, one can make additions or subtractions at $\Gamma$ or $K$ and promote electrons either from $K_{\mathrm{v}} \rightarrow \Gamma_{\mathrm{c}}$ or from $K_{\mathrm{v}} \rightarrow K_{\mathrm{c}}$. In a general supercell, however, one can choose the supercell Bloch vector $\mathbf{k}_{\mathrm{s}}$ so that the orbitals at $\Gamma$ are present in the Slater wave function, or so that the orbitals at $K$ are present, but not both at the same time. The quasiparticle gap from $K_{\mathrm{v}} \rightarrow \Gamma_{\mathrm{c}}$ can always be calculated for a given supercell by determining the CBM and VBM using two different values of $\mathbf{k}_{\mathrm{s}}$. Similar comments apply in the bulk case. With the optimal (nondiagonal) supercell matrices for $N_{\mathrm{P}}=\operatorname{det}(S)=$ 18 or 36 primitive cells we are unable to include $\Gamma$ and $K$ simultaneously in the grid of $\mathbf{k}$ vectors. This prevents calculation of the $\Gamma_{\mathrm{v}} \rightarrow K_{\mathrm{c}}$ excitonic gap in these supercells. We have instead calculated the bulk $\Gamma_{\mathrm{v}} \rightarrow K_{\mathrm{c}}$ excitonic gaps in supercells defined by diagonal supercell matrices $S\left(N_{\mathrm{P}}=\right.$ $18)=\operatorname{diag}(3,3,2)$ and $S\left(N_{\mathrm{P}}=36\right)=\operatorname{diag}(3,3,4)$.

Now, let us consider "long-range" finite-size errors in the energy gaps in periodic supercells. Adding a single electron to or removing a single electron from a periodic simulation supercell results in the creation of an unwanted lattice of quasiparticles at the set of supercell lattice points [40]. The leading-order systematic finite-size error in the quasiparticle bands is therefore $v_{\mathrm{M}} / 2$, where $v_{\mathrm{M}}$ is the screened Madelung constant [69] of the supercell. The situation is qualitatively similar to that encountered in $a b$ initio simulations of charged defects [70]. Following the notation of Sec. II C 1, a finitesize-corrected expression for an unoccupied energy band is $\mathcal{E}_{i}^{\prime}(\mathbf{k})=\left[E^{+}(\mathbf{k}, i)-v_{\mathrm{M}} / 2\right]-E^{\mathrm{GS}}$. In a similar fashion, when one creates a lattice of holes by removing an electron from a periodic supercell, a finite-size-corrected expression for an occupied energy band is $\mathcal{E}_{i}^{\prime}(\mathbf{k})=E^{\mathrm{GS}}-\left[E^{-}(\mathbf{k}, i)-v_{\mathrm{M}} / 2\right]$. The finite-size-corrected quasiparticle band gap is therefore

$$
\Delta_{\mathrm{qp}}^{\prime}=\mathcal{E}_{\mathrm{CBM}}^{\prime}-\mathcal{E}_{\mathrm{VBM}}^{\prime}=E_{\mathrm{CBM}}^{+}+E_{\mathrm{VBM}}^{-}-2 E^{\mathrm{GS}}-v_{\mathrm{M}} .
$$


In an hBN monolayer, in-plane screening modifies the form of the Coulomb interaction between charges. The screened interaction is approximately of Keldysh form [71]. Including the relative permittivity $\epsilon$ of the surrounding medium, the Keldysh interaction in reciprocal space is $v(k)=2 \pi /[\epsilon k(1+$ $\left.r_{*} k\right)$ ], where $r_{*}$ is the ratio of the in-plane susceptibility of the layer to the permittivity of the surrounding medium. For the monolayer the leading-order finite-size error in the quasiparticle gap is the Madelung constant $v_{\mathrm{M}}$ of the supercell evaluated using the Keldysh interaction [40]. In small supercells, the Keldysh interaction between nearby periodic images varies logarithmically with $r$ and the Madelung constant is almost independent of system size; however, once the supercell size significantly exceeds $r_{*}$, the Keldysh interaction reduces to the Coulomb $1 / r$ form and the Madelung constant falls off as the reciprocal of the linear size of the supercell.

The parameter $r_{*}$ may be estimated using Eq. (S7) in the Supplemental Material of Ref. [72]. For free-standing monolayer hBN, $r_{*} \approx c\left(\epsilon_{\|}-1\right) / 4$, where $c$ is the out-of-plane lattice parameter of bulk hBN, $\epsilon_{\|}$is the in-plane component of the high-frequency permittivity tensor, and we have included an extra factor of $\frac{1}{2}$ due to the fact that there are two layers per bulk hBN primitive cell. The lattice parameter is measured to be $c=6.6612 \AA$ [73], while DFT-PBE calculations predict that $\epsilon_{\|} \approx 4.69$, giving $r_{*} \approx 6.14 \AA$. Unfortunately, the supercell sizes used in this work are comparable in size to $r_{*}$. For bulk hBN the $r_{*}$ value is smaller by a factor $\sqrt{\epsilon_{\|} \epsilon_{z}}$, where $\epsilon_{z}$ is the out-of-plane component of the permittivity tensor [72]. Using the DFT-PBE high-frequency out-of-plane permittivity $\epsilon_{z}=2.65$ gives $r_{*}^{\text {bulk }}=1.74 \AA$ for bulk hBN. Our bulk hBN supercells are sufficiently large that the interaction between periodic images can be assumed to be of Coulomb $1 / r$ form; nevertheless, the strong anisotropy of the dielectric screening must be taken into account in the evaluation of the screened Madelung constant [74] $v_{\mathrm{M}}$.

The remaining systematic finite-size effects in the quasiparticle gap are primarily due to charge-quadrupole image interactions and fall off as $a_{\mathrm{s}}^{-2}$ when $a_{\mathrm{s}} \leqslant r_{*}$ and as $a_{\mathrm{s}}^{-3}$ when $a_{\mathrm{s}} \gg r_{*}$, where $a_{\mathrm{s}}$ is the in-plane linear size of the supercell [40]. There are also oscillatory, quasirandom errors with a slowly decaying envelope as a function of system size due to long-range oscillations in the pair-correlation function being forced to be commensurate with the supercell (see Figs. 5 and 9). We remove the remaining finite-size errors by extrapolating the Madelung-corrected quasiparticle gaps in supercells of nine or more primitive cells to infinite system size, assuming the finite-size error decays as $a_{\mathrm{s}}^{-2}$ in monolayer $\mathrm{hBN}$ and as $a_{\mathrm{s}}^{-3}$ in bulk hBN (i.e., as $N_{\mathrm{P}}^{-1}$ in both cases). Since the quasirandom finite-size errors dominate the QMC statistical error bars, we do not weight our data by the QMC error bars [40].

Note that the uncorrected quasiparticle gap calculated in a finite supercell may be smaller than the excitonic gap in that supercell due to a negative Madelung constant, as can be seen in Tables I and II. This is simply an artifact of the use of periodic boundary conditions and the Ewald interaction, and the effect disappears in the thermodynamic limit of infinite system size, where the excitonic gap must always be less than or equal to the quasiparticle gap due to the attractive interaction between electrons and holes.
TABLE III. Effective masses $m^{*}$ for the $\Gamma_{\mathrm{c}}, K_{\mathrm{c}}$, and $K_{\mathrm{v}}$ bands from DFT-LDA, DFT-PBE, and DFT-HSE06 calculations.

\begin{tabular}{lccc}
\hline \hline & \multicolumn{3}{c}{$m^{*}($ a.u. $)$} \\
\cline { 2 - 4 } Functional & $\Gamma_{\mathrm{c}}$ & $K_{\mathrm{c}}$ & $K_{\mathrm{v}}$ \\
\hline LDA & 0.96 & 0.89 & 0.61 \\
PBE & 0.95 & 0.90 & 0.63 \\
HSE06 & 0.98 & 1.07 & 0.63 \\
\hline \hline
\end{tabular}

Finite-size effects in DMC excitonic gaps may arise from the confinement of a neutral exciton in a periodic simulation supercell. Once the supercell size significantly exceeds the size of the exciton, the exciton wave function is exponentially localized within the supercell; however, power-law finite-size effects in the exciton binding energy remain due to the difference between the screened Coulomb interaction in a finite, periodic supercell and in an infinite system. The length scale of an exciton under the Keldysh interaction is $r_{0}=\sqrt{r_{*} /(2 \mu)}$, where $\mu$ is the electron-hole reduced mass [72,75]. Using the DFT-HSE06 effective masses in Table III together with the $r_{*}$ value estimated above, we find the sizes of both the $K_{\mathrm{v}} \rightarrow \Gamma_{\mathrm{c}}$ and $K_{\mathrm{v}} \rightarrow K_{\mathrm{c}}$ excitons in monolayer hBN to be $r_{0} \approx 3 \AA$, which is only slightly larger than the lattice constant. Hence, all our simulation supercells are large enough to contain the excitons, and so the remaining finite-size effects are due to instantaneous dipole-dipole interactions between identical images, evaluated with the Keldysh interaction in the case of the monolayer. On the other hand, the fact that the exciton radius is comparable with the lattice constant implies that we are at the limit of the validity of the effective-mass model of excitons and it may not fully account for finite-size effects in gaps; nevertheless, this model provides us with the best available framework for understanding systematic finite-size effects. In supercells with $a_{\mathrm{s}} \leqslant r_{*}$, the leading-order systematic finitesize effects in the excitonic gap go as $a_{\mathrm{s}}^{-2}$; for supercells with $a_{\mathrm{s}} \gg r_{*}$, the finite-size effects go as $a_{\mathrm{s}}^{-3}$. We therefore extrapolate our uncorrected excitonic gaps in the same way that we extrapolate our Madelung-corrected quasiparticle gaps to infinite system size, i.e., assuming the errors go as $N_{\mathrm{P}}^{-1}$ for both bulk and monolayer. Again, we do not weight our data by the QMC error bars since the quasirandom finite-size effects dominate the QMC error bars.

Our DMC gaps against system size are presented and discussed in Sec. III C 1.

\section{Vibrational contribution}

We calculated the vibrational contribution to the quasiparticle band gap arising from the electron-phonon interaction at temperature $T$ within the Born-Oppenheimer approximation as

$$
\Delta_{\mathrm{qp}}(T)=\frac{1}{\mathcal{Z}} \sum_{\mathbf{s}}\left\langle\Phi_{\mathbf{s}}(\mathbf{u})\left|\Delta_{\mathrm{qp}}(\mathbf{u})\right| \Phi_{\mathbf{s}}(\mathbf{u})\right\rangle e^{-E_{\mathbf{s}} /\left(k_{\mathrm{B}} T\right)},
$$

where the harmonic vibrational wave function $\left|\Phi_{\mathbf{s}}(\mathbf{u})\right\rangle$ in state $\mathbf{s}$ has energy $E_{\mathbf{s}}, \mathbf{u}=\left\{u_{v \mathbf{q}}\right\}$ is a collective coordinate for all the nuclei written in terms of normal modes of vibration 
$(v, \mathbf{q}), \mathcal{Z}=\sum_{\mathrm{s}} e^{\left.-E_{\mathrm{s}} / k_{\mathrm{B}} T\right)}$ is the partition function, and $k_{\mathrm{B}}$ is Boltzmann's constant.

We evaluated Eq. (5) using two complementary methods recently reviewed in Ref. [76]. The first relies on a stochastic Monte Carlo sampling of the vibrational density over $M$ points:

$$
\Delta_{\mathrm{qp}}^{\mathrm{MC}}(T)=\frac{1}{M} \sum_{i=1}^{M} \Delta_{\mathrm{qp}}\left(\mathbf{u}_{i}\right),
$$

where configurations $\mathbf{u}_{i}$ are distributed according to the nuclear density. This approach enables the inclusion of the electron-phonon interaction at all orders at the expense of using large diagonal supercell matrices, and in practice we use thermal lines to accelerate the sampling [77]. The second approach relies on a second-order expansion of the dependence of $\Delta_{\mathrm{qp}}(\mathbf{u})$ on the mode amplitudes $\mathbf{u}$, which leads to a particularly simple quadratic approximation

$$
\Delta_{\mathrm{qp}}^{\mathrm{quad}}(T)=\Delta_{\mathrm{qp}}+\frac{1}{N_{\mathbf{q}}} \sum_{\mathbf{q}, v} \frac{1}{\omega_{\mathbf{q} v}} \frac{\partial^{2} \Delta_{\mathrm{qp}}}{\partial u_{\mathbf{q} v}^{2}}\left[\frac{1}{2}+n_{\mathrm{B}}\left(\omega_{\mathbf{q} v}, T\right)\right],
$$

where $n_{\mathrm{B}}\left(\omega_{\mathbf{q} v}, T\right)$ is a Bose-Einstein factor. This expression can be efficiently evaluated using nondiagonal supercell matrices [66] at the expense of neglecting higher-order terms in the electron-phonon interaction. Overall, Eq. (7) enables the convergence of the calculations with respect to supercell size (or equivalently q-point grid density), whereas Eq. (6) enables the inclusion of higher-order terms, which have been found to provide important contributions in a range of materials $[78,79]$.

All our vibrational calculations were performed using the PBE functional, an energy cutoff of $700 \mathrm{eV}$, and a k-point spacing of $2 \pi \times 0.025 \AA^{-1}$ to sample the electronic Brillouin zone. The results show slow convergence with respect to the q-point grid size: the vibrational correction to the quasiparticle gap at $300 \mathrm{~K}$ using the expression in Eq. (7) converges to values better than $0.05 \mathrm{eV}$ using a grid size of $32 \times 32 \mathbf{q}$ points for the monolayer, and using a grid size of $16 \times 16 \times 16$ for the bulk. We also tested the inclusion of van der Waals dispersion corrections in the bulk calculations using the Tkatchenko-Scheffler scheme [80] but found differences smaller than $0.01 \mathrm{eV}$ compared to the calculations without dispersion corrections. Using Eq. (6) instead of Eq. (7) leads to a significant enhancement to the vibrational correction to the quasiparticle gap. However, calculations using Eq. (6) are restricted to smaller q-point grid sizes, and therefore our final results were estimated by using the q-point converged results obtained with Eq. (7) and adding a correction equal to $\Delta_{\mathrm{qp}}^{\mathrm{MC}}(T)-\Delta_{\mathrm{qp}}^{\mathrm{quad}}(T)$ evaluated at the largest q-point grid size feasible within the Monte Carlo method, which is $8 \times 8$ for the monolayer and $4 \times 4 \times 4$ for the bulk.

\section{RESULTS}

\section{A. Lattice parameter and dynamical stability}

The lattice parameters obtained in DFT-LDA, DFT-PBE, and DFT-HSE06 calculations are $a=2.491,2.512$, and 2.45 $\AA$, respectively, which may be compared with the bulk lattice

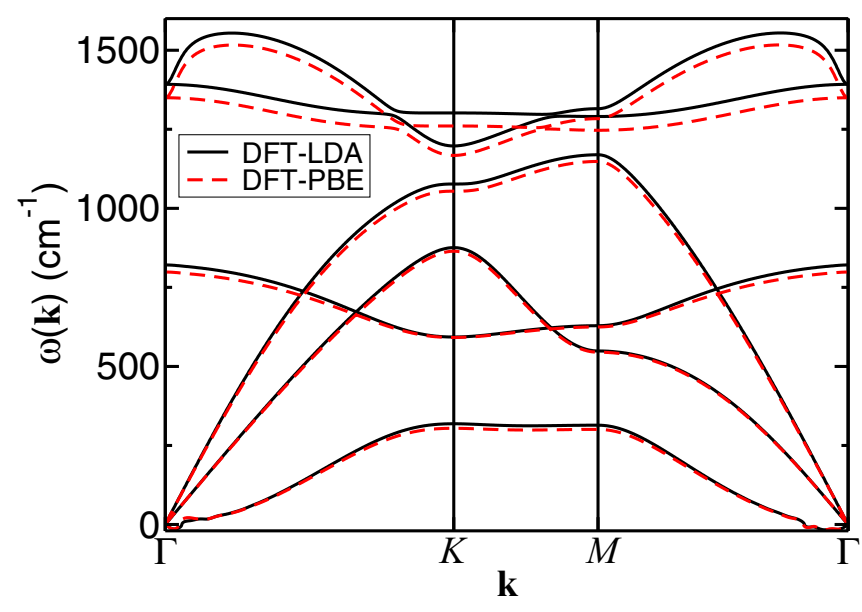

FIG. 1. DFT-LDA and DFT-PBE phonon dispersion curves for monolayer hBN.

parameter $a=2.5040 \AA[73]$ and the lattice parameter $2.5 \AA$ measured in a thin film of hBN [26]. Our DFT-PBE lattice parameter is in good agreement with a previously published result, $a=2.51 \AA$ [81]. We have used the DFT-PBE lattice parameter $a=2.512 \AA$ in all our QMC calculations. The partial charge of each boron atom is 0.83 according to Mulliken population analysis of the DFT orbitals [82] and 0.21 a.u. according to Hirshfeld analysis of the charge density [83]. The partial charges predicted by the LDA and PBE functionals agree.

The DFT-LDA and DFT-PBE phonon dispersion curves of hBN are shown in Fig. 1. The calculations appear to predict a small region of dynamical instability in the flexural acoustic branch about the $\Gamma$ point. Such regions of instability around $\Gamma$ are a common feature in first-principles latticedynamics calculations for 2D materials, including graphene, molybdenum disulfide, and indium and gallium chalcogenides [84]. We observe that (i) the region of instability occurs in both finite-displacement (supercell) calculations and in density functional perturbation theory calculations; (ii) the region of instability depends sensitively on every simulation parameter (basis set, k-point sampling, supercell size in finitedisplacement calculations, exchange-correlation functional, pseudopotential, and artificial periodicity); (iii) the size of the instability is the same as the amount by which the acoustic branches miss zero at $\Gamma$ if Newton's third law is not imposed on the force constants; and (iv) the region of instability remains even if the layer is put under tension by increasing the lattice parameter slightly. To minimize the effects of longitudinal/transverse optic-mode splitting in our three-dimensionally periodic calculations, we choose the $z$ component of the wave vector to be $\pi / L$, where $L$ is the artificial periodicity [85]. Our results are in good agreement with the phonon dispersion curves obtained by Wirtz et al. [85]. An analysis of the Raman activity of phonon modes is given in Ref. [86].

\section{B. DFT electronic band structure and effective masses}

The DFT-LDA, DFT-PBE, and DFT-HSE06 band structures of monolayer and bulk hBN are shown in Figs. 2 and 3, 


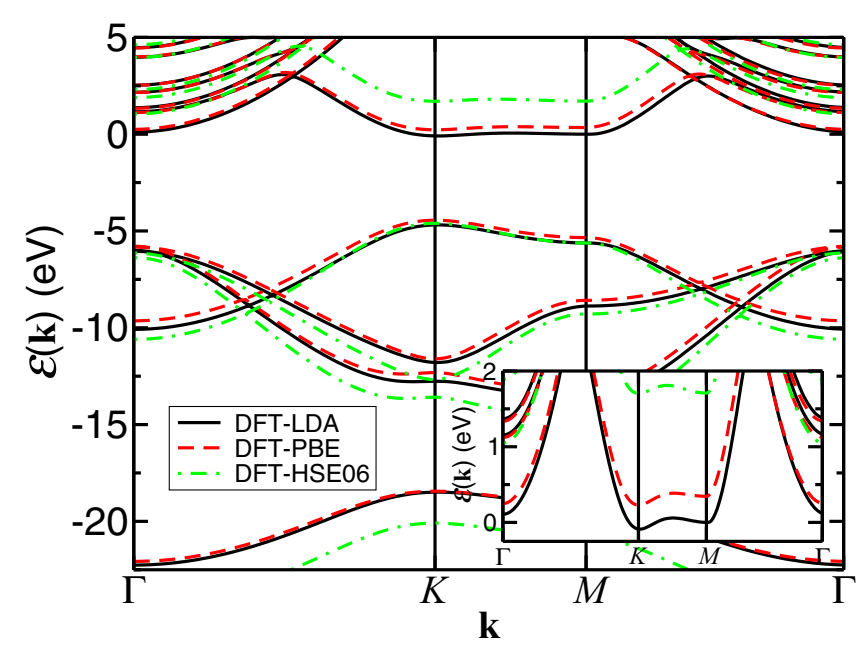

FIG. 2. DFT-LDA, DFT-PBE, and DFT-HSE06 electronic bandstructure plots for monolayer hBN. The zero of energy is set to the Fermi energy. The inset shows the energy range around the CBM in greater detail.

respectively. In the case of the monolayer, we fitted

$$
\begin{aligned}
\mathcal{E}_{\mathrm{c}, \mathrm{v}}(\mathbf{q})= & \mathcal{E}_{K_{\mathrm{c}, \mathrm{v}}} \pm \frac{q^{2}}{2 m_{K_{\mathrm{c}, \mathrm{v}}^{*}}^{*}}+A_{\mathrm{c}, \mathrm{v}} q^{4}+B_{\mathrm{c}, \mathrm{v}} q^{6} \\
& +C_{\mathrm{c}, \mathrm{v}} q^{6} \cos (6 \theta)+D_{\mathrm{c}, \mathrm{v}} q^{3} \cos (3 \theta) \\
& +E_{\mathrm{c}, \mathrm{v}} q^{5} \cos (3 \theta)
\end{aligned}
$$

where $\mathcal{E}_{K_{\mathrm{c}, \mathrm{v}}}, m_{K_{\mathrm{c}, \mathrm{v}}}^{*}, A_{\mathrm{c}, \mathrm{v}}, B_{\mathrm{c}, \mathrm{v}}, C_{\mathrm{c}, \mathrm{v}}, D_{\mathrm{c}, \mathrm{v}}$, and $E_{\mathrm{c}, \mathrm{v}}$ are fitting parameters, to the conduction and valence bands within a circle of radius $6 \%$ of the $\Gamma-M$ distance around the $K$ point. $\mathbf{q}$ is the wave vector relative to the $K$ point, and $\theta$ is the polar angle of $\mathbf{q}$. The second term is positive for the conduction band and negative for the valence band, so that $m_{K_{\mathrm{c}}}^{*}$ and $m_{K_{\mathrm{v}}}^{*}$ are the electron and hole effective masses. The root-meansquare (rms) residual over the fitting area is less than $0.2 \mathrm{meV}$

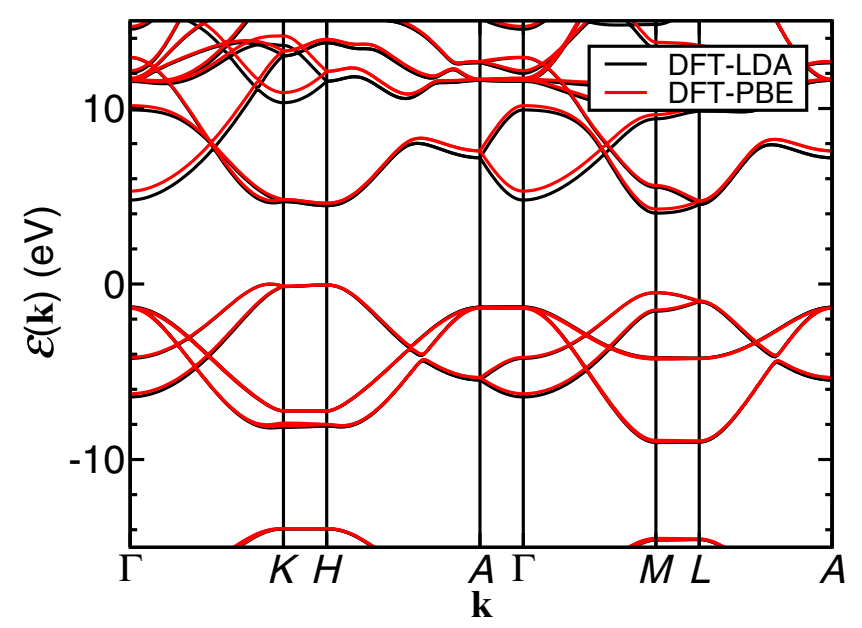

FIG. 3. DFT-LDA and DFT-PBE electronic band-structure plots for bulk hBN. The zero of energy is set to the Fermi energy.
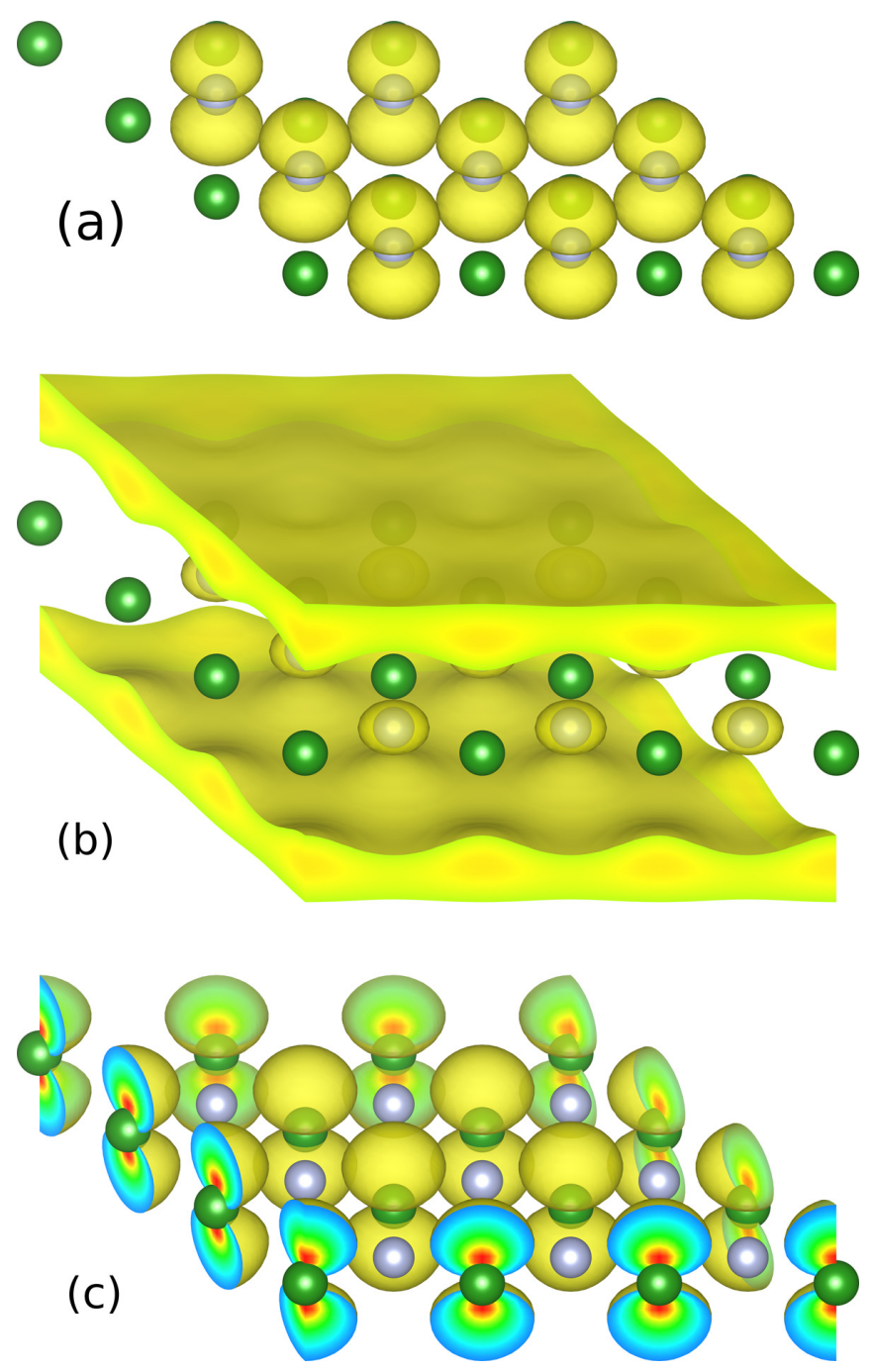

FIG. 4. DFT-HSE06 charge densities of (a) the valence-band maximum at $K_{\mathrm{v}}$, (b) the conduction-band minimum at $\Gamma_{\mathrm{c}}$, and (c) the conduction-band minimum at $K_{\mathrm{c}}$ for monolayer $\mathrm{hBN}$. The green spheres show the boron atoms, while the white spheres are nitrogen atoms. The charge densities were obtained using an artificial periodicity of $21.2 \AA$ in the out-of-plane direction, a $15 \times 15$ MonkhorstPack k-point grid, DFT norm-conserving pseudopotentials, and a plane-wave cutoff energy of $680 \mathrm{eV}$.

in each case. We fitted

$$
\mathcal{E}_{\mathrm{c}}(\mathbf{k})=\mathcal{E}_{\Gamma_{\mathrm{c}}}+\frac{k^{2}}{2 m_{\Gamma_{\mathrm{c}}}^{*}}+A^{\prime} k^{4}+B^{\prime} k^{6}+C^{\prime} k^{6} \cos (6 \theta),
$$

where $k=|\mathbf{k}|, \theta$ is the polar angle of $\mathbf{k}$, and $\mathcal{E}_{\Gamma_{\mathrm{c}}}, m_{\Gamma_{\mathrm{c}}}^{*}, A^{\prime}, B^{\prime}$, and $C^{\prime}$ are fitting parameters, to the conduction band within a circle of radius $40 \%$ of the $\Gamma-M$ distance about $\Gamma$. The rms residual over this area is less than $0.2 \mathrm{meV}$ in each case. It is clearly much easier to represent the band over a large area around $\Gamma$ than around $K$. The fitted effective masses in Eqs. (8) and (9) are reported in Table III. It was verified that the effective masses are unchanged to the reported precision when the radius of the circle used for the fit is reduced.

The DFT charge density of the conduction-band minimum at $\Gamma_{\mathrm{c}}$ consists of two delocalized, free-electron-like regions on 


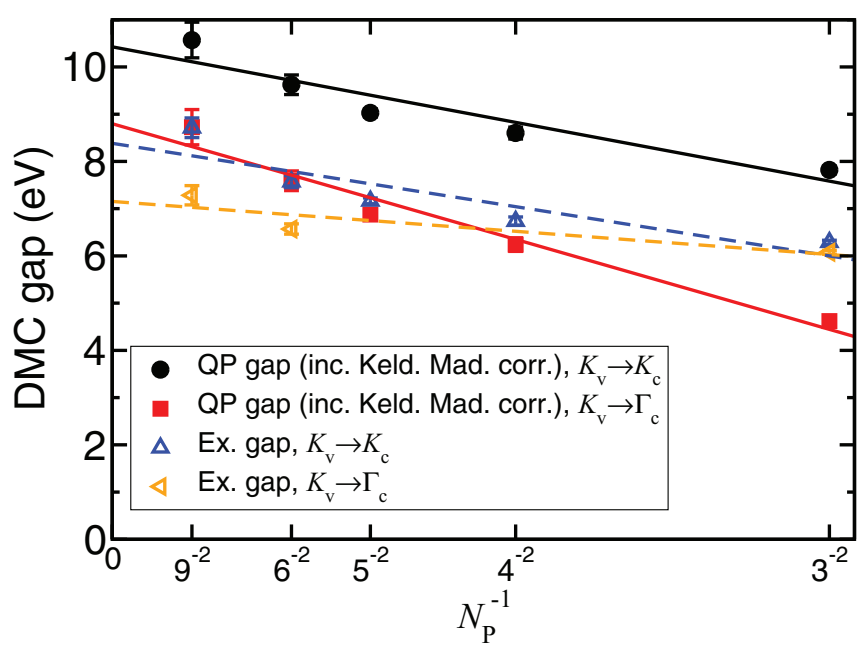

FIG. 5. SJ-DMC quasiparticle (QP) and excitonic gaps of monolayer hBN against $N_{\mathrm{P}}^{-1}$, where $N_{\mathrm{P}}$ is the number of primitive cells in the supercell. The quasiparticle gaps include the Madelung correction given in Eq. (4).

either side of the hBN layer, whereas the charge density for the conduction-band minimum at $K_{\mathrm{c}}$ is localized on the boron atoms (see Fig. 4). This is consistent with the observation that the conduction band at $\Gamma$ is nearly parabolic with an effective mass close to the bare electron mass. The orbital charge densities are qualitatively similar in the monolayer and in the bulk.

\section{Energy-gap results}

\section{Finite-size effects in the DMC band gap}

The SJ-DMC quasiparticle and excitonic band gaps (both $K_{\mathrm{v}} \rightarrow K_{\mathrm{c}}$ and $K_{\mathrm{v}} \rightarrow \Gamma_{\mathrm{c}}$ ) are plotted against system size in Fig. 5. The quasiparticle gaps include the correction shown in Eq. (4). Systematic finite-size effects in the $K_{\mathrm{v}} \rightarrow \Gamma_{\mathrm{c}}$ and $K_{\mathrm{v}} \rightarrow K_{\mathrm{c}}$ excitonic gaps are very much smaller than systematic finite-size errors in the uncorrected quasiparticle gaps. On the other hand, the quasirandom finite-size noise in both types of gap has an amplitude of about $0.5 \mathrm{eV}$ over the range of supercells studied.

\section{Nature and size of the gap in the thermodynamic limit}

Our results for the electronic band gaps are given in Table IV. The error bars on our QMC gaps are determined by the quasirandom finite-size noise discussed in Secs. II C 7 and III C 1. The SJ-DMC quasiparticle gap of monolayer hBN is indirect $\left(K_{\mathrm{V}} \rightarrow \Gamma_{\mathrm{c}}\right)$ and is of magnitude $8.8(3) \mathrm{eV}$, which is considerably enhanced with respect to the gap in the bulk, and is also significantly higher than the gap predicted by our $G W_{0}$ calculations $(7.72 \mathrm{eV})$.

In Fig. 6 we compare the electronic band structure predicted by the two levels of $G W$ theory and DFT-PBE calculations (top panel), and we plot the $G W_{0}$-BSE absorption spectrum (bottom panel). The exciton binding energy is extracted by comparing the BSE optical absorption spectrum with its random-phase-approximation counterpart, in which electron-hole interactions are neglected. Our single-shot $G_{0} W_{0}$ quasiparticle gaps are significantly smaller than the SJ-DMC gaps, by about 1.4-2.5 eV. The partially self-consistent $G W_{0}$ quasiparticle gaps are somewhat larger, but are still 1.1-2.2 eV smaller than the SJ-DMC quasiparticle gaps. The exciton binding energies obtained using first-principles $G W_{0}$-BSE and SJ-DMC calculations are in reasonable agreement with the exciton binding energies of $2.38 \mathrm{eV}\left(\Gamma_{\mathrm{v}} \rightarrow K_{\mathrm{c}}\right)$ and $2.41 \mathrm{eV}$ $\left(K_{\mathrm{v}} \rightarrow K_{\mathrm{c}}\right)$ obtained using an effective-mass model [75] of an electron and a hole interacting via the Keldysh interaction with the effective masses in Table III and the $r_{*}$ parameter estimated in Sec. II C 7.

DFT-LDA and DFT-PBE band-structure calculations are qualitatively incorrect for monolayer $\mathrm{hBN}$ : they predict the gap to be direct $\left(K_{\mathrm{v}} \rightarrow K_{\mathrm{c}}\right)$. DFT-HSE06 and $G W$ calculations show that the conduction-band energies at $K_{\mathrm{c}}$ and $M_{\mathrm{c}}$ are similar [87], but that the CBM lies at $\Gamma_{\mathrm{c}}$, in agreement with SJ-DMC.

We find the gap of monolayer $\mathrm{hBN}$ to be indirect, with the CBM lying at the $\Gamma_{\mathrm{c}}$ point, although recent experiments indicate a direct gap at the $K$ point of the Brillouin zone [34]. Part of the reason for the discrepancy is that those experiments studied hBN on a graphite substrate; however, the delocalized nature of the (nearly free) $\mathrm{CBM}$ state at $\Gamma_{\mathrm{c}}$ may also have consequences for optical absorption experiments. Electrons with small in-plane momentum experience the hBN monolayer as an attractive $\delta$-function-like potential, always supporting one bound state. This weakly bound state is potentially sensitive to perturbations caused by substrates or other aspects of the material environment. We have investigated the behavior of

TABLE IV. Static-nucleus quasiparticle and excitonic gaps for monolayer hBN, calculated by different methods. Our DFT calculations indicate that vibrational effects lead to a renormalization of the static-nucleus gaps by $-0.73 \mathrm{eV}$ at $300 \mathrm{~K}$.

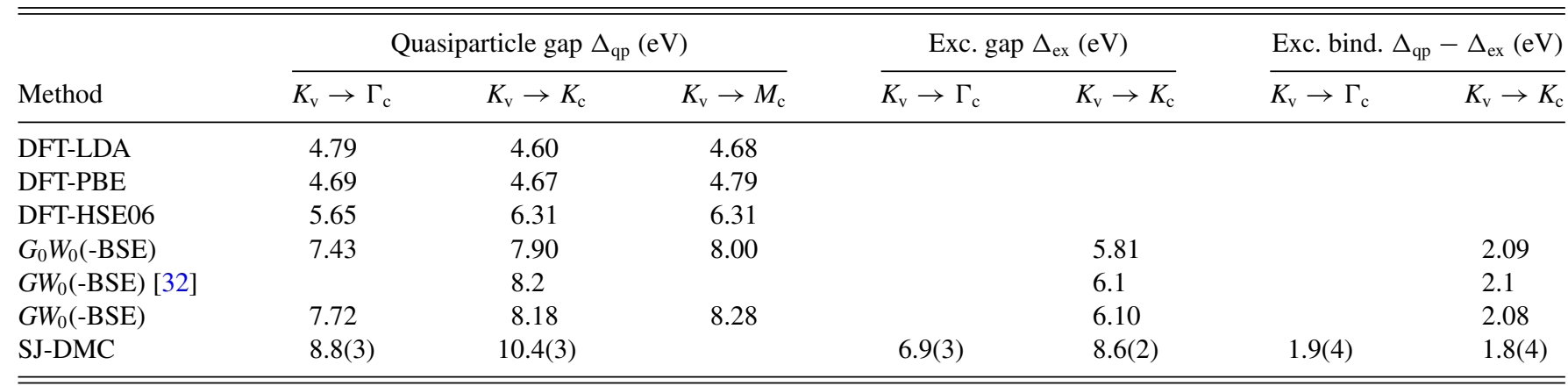



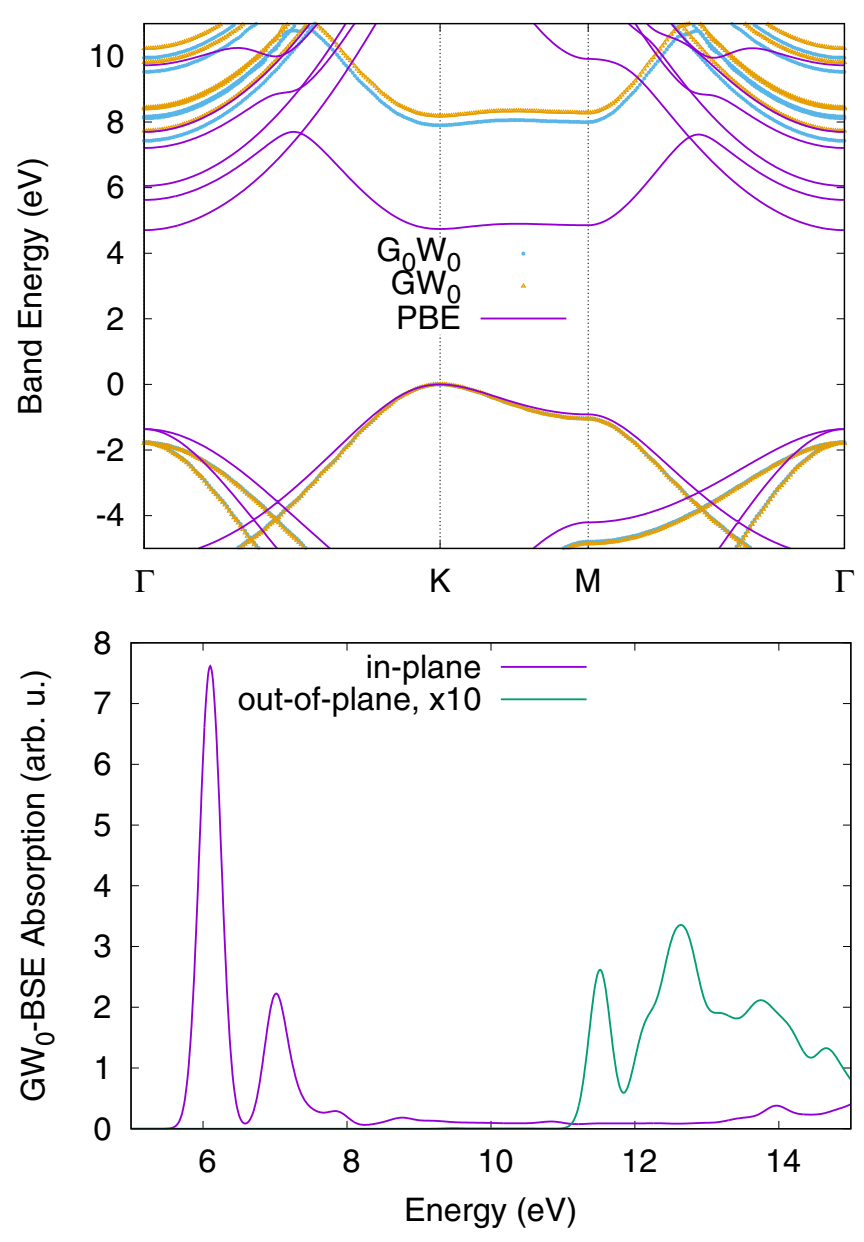

FIG. 6. Electronic band structure of monolayer hBN, comparing DFT-PBE with $G W$ theory at the single-shot $\left(G_{0} W_{0}\right)$ and partially self-consistent $\left(G W_{0}\right)$ level (top panel). $G W_{0}$-BSE optical absorption spectrum of monolayer hBN for in-plane and out-of-plane polarization (bottom panel).

the conduction band at $\Gamma_{\mathrm{c}}$ in bulk hBN as the out-of-plane lattice parameter $c$ is increased, describing the crossover from bulk to isolated monolayer. In Fig. 7, we plot the normalized DFT-PBE charge density of the state at $\Gamma_{\mathrm{c}}$ along a line through the unit cell, moving through a boron atom at $z / c=0.25$ and

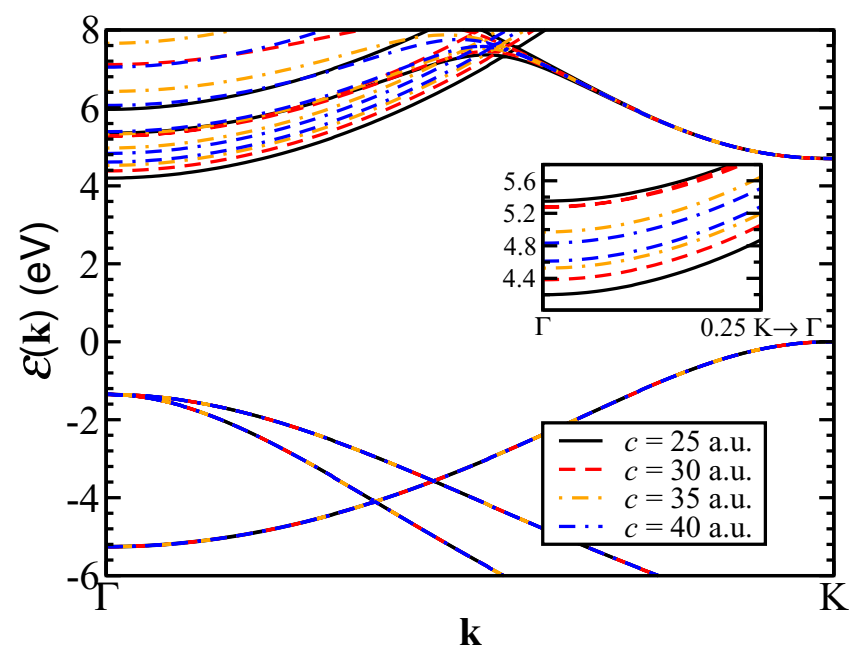

FIG. 8. DFT-PBE bulk hBN band structure at four large values of the lattice parameter $c$. The inset displays a closeup of the two near-degenerate states at $\Gamma_{\mathrm{c}}$.

through a nitrogen atom at $z / c=0.75$. In Fig. 8 we plot the DFT-PBE band structure along the $\Gamma \rightarrow \mathrm{K}$ line. While all other states are well converged with respect to $c$, including the state at $K_{\mathrm{c}}$, the state at $\Gamma_{\mathrm{c}}$ remains relatively sensitive to the particular choice of $c$. In the inset to Fig. 8, the two lowestlying conduction states have been retained for clarity, and this sensitivity is made very clear. The expected trend in the energy of the two near-degenerate conduction states originating from each monolayer is observed, and as $c$ increases, the energy splitting of these two states reduces.

\section{Vibrational renormalization of the band structure}

Using a combination of the quadratic and stochastic approaches as described in Sec. IID, we obtain a vibrational renormalization of the minimum band gap $K_{\mathrm{v}} \rightarrow \Gamma_{\mathrm{c}}$ of monolayer hBN of $-0.56 \mathrm{eV}$ at $0 \mathrm{~K}$. This zero-temperature correction arises purely from quantum zero-point motion, which has a strong effect in a system like hBN containing light elements, and is similar in size to that calculated for diamond [88-90]. Thermal motion further renormalizes the band gap, resulting in a vibrational correction of $-0.73 \mathrm{eV}$ at $300 \mathrm{~K}$.
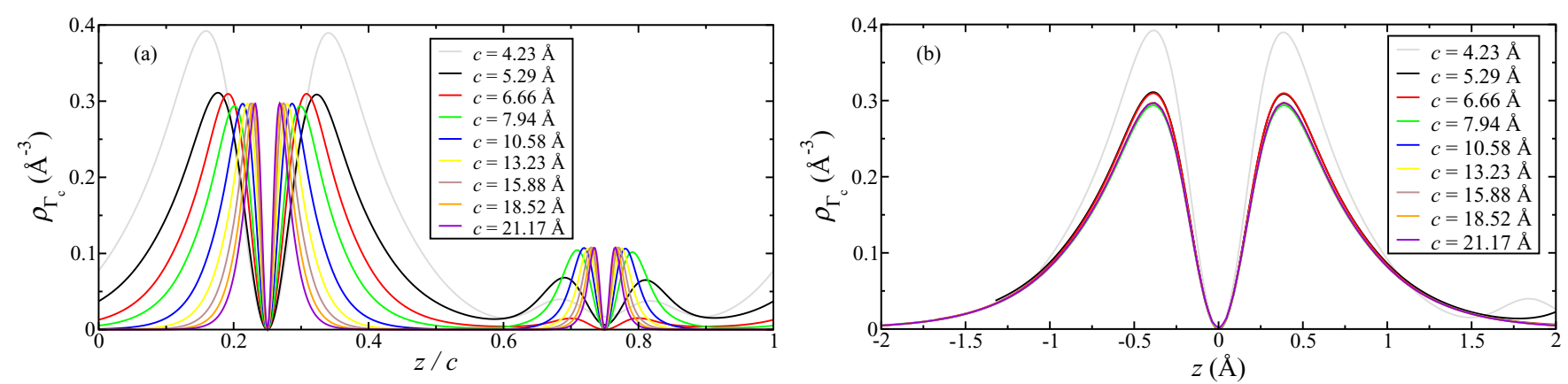

FIG. 7. DFT-PBE charge density of the state at $\Gamma_{\mathrm{c}}$ as a function of lattice parameter $c$ for bulk hBN. $c=12.5878$ a.u. is the experimental lattice parameter. (a), (b) Show the density against fractional and absolute $z$ coordinates, respectively. The charge density is plotted along a straight line in the $z$ direction, passing through a boron atom at $z / c=0.25$ and a nitrogen atom at $z / c=0.75$. At large $c$, the $\mathrm{CBM}$ at $\Gamma_{\mathrm{c}}$ is an arbitrary linear combination of the degenerate monolayer CBMs. 
TABLE V. Static-nucleus quasiparticle and excitonic gaps for bulk hBN, determined by different methods, compared with experimental results. Our DFT vibrational-renormalization calculations indicate that the static-nucleus gaps should be renormalized by $-0.40 \mathrm{eV}$ at $300 \mathrm{~K}$. Where references are not given, the results are from this work. An asterisk (*) denotes the SJ-DMC energy gap from $K_{\mathrm{v}} \rightarrow M_{\mathrm{c}}$.

\begin{tabular}{|c|c|c|c|c|c|c|c|c|c|c|}
\hline Method & \multicolumn{6}{|c|}{ Quasiparticle gap $\Delta_{\mathrm{qp}}(\mathrm{eV})$} & \multicolumn{4}{|c|}{ Excitonic gap $\Delta_{\mathrm{ex}}(\mathrm{eV})$} \\
\hline DFT-PBE & 6.65 & 4.76 & 5.42 & 4.94 & 5.78 & 4.28 & & & & \\
\hline DFT-HSE06 & 8.01 & 6.09 & 6.54 & 6.33 & 6.95 & 5.55 & & & & \\
\hline$G_{0} W_{0}$ & 7.3 & 7.0 & & 9.7 & 6.1 & 5.4 & & & & \\
\hline SJ-DMC & $10.1(2)$ & & $8.5(2)$ & $9.06(8)$ & & $7.96(9)^{*}$ & $9.2(2)$ & $8.2(5)$ & $8.3(1)$ & \\
\hline Expt. [25,27] & & & & & & $5.971,6.08$ & & & & $5.822,5.955$ \\
\hline
\end{tabular}

Our results for the $K_{\mathrm{v}} \rightarrow K_{\mathrm{c}}$ gap show a zero-point renormalization of the band gap of $-0.54 \mathrm{eV}$, which increases to $-0.73 \mathrm{eV}$ at $300 \mathrm{~K}$. The similar corrections for the $K_{\mathrm{v}} \rightarrow \Gamma_{\mathrm{c}}$ and $K_{\mathrm{v}} \rightarrow K_{\mathrm{c}}$ gaps suggest that vibrational corrections to the gap are largely uniform across the Brillouin zone.

\section{Bulk hBN}

As a test of the accuracy of our methods, we have calculated the quasiparticle and excitonic gaps of bulk hBN between various high-symmetry points in the Brillouin zone with the QMC and $G W$ methods. Our QMC calculations are identical to those performed for the monolayer, save for the use of the experimental geometry (lattice parameters $a=$ $2.504 \AA$ and $c=6.6612 \AA$ ) [73], and the use of the "T-move" scheme, which reduces pseudopotential locality approximation errors [91-93].

Our QMC results are given in Table V, with error bars determined as discussed in Sec. II C 7. Our raw gap data are plotted against system size in Fig. 9. We find that quasirandom finite-size effects are much more prominent in the bulk than in the monolayer. This could be partially due to the lack of geometrical similarity of the supercells studied, leading to nonsystematic behavior in the charge-quadrupole finite-size effect. Our $G W$ results for bulk hBN are also shown in Table V. Here, we find that the quasiparticle gaps evaluated with SJ-DMC are somewhat larger than those predicted by $G W$ calculations, just as they are in the monolayer.

The SJ-DMC $K_{\mathrm{v}} \rightarrow K_{\mathrm{c}}$ exciton binding energy of bulk $\mathrm{hBN}$, which is corrected by the subtraction of the screened Madelung constant and then extrapolated against $N_{\mathrm{P}}^{-1}$ to infinite system size [40], is $0.8(1) \mathrm{eV}$. This is consistent with the range of $G W$-BSE values, and is significantly smaller than the monolayer exciton binding energy, as one would expect. The $K_{\mathrm{V}} \rightarrow \Gamma_{\mathrm{c}}$ exciton binding is $0.3(5) \mathrm{eV}$, which is smaller than the statistical error bars.

The SJ-DMC $K_{\mathrm{v}} \rightarrow M_{\mathrm{c}}$ quasiparticle gap is $7.96(9) \mathrm{eV}$. The VBM in bulk hBN is near the $K$ point, while the CBM is at or near the $M$ point [27]. Allowing for our calculated zerotemperature vibrational correction in bulk hBN of $-0.35 \mathrm{eV}$ (which increases to $-0.40 \mathrm{eV}$ at $300 \mathrm{~K}$ ), the SJ-DMC quasiparticle gap appears to overestimate the experimental gap of around $6 \mathrm{eV}$ significantly. As a probe of this discrepancy, we have considered (in the $N_{\mathrm{P}}=9$ supercell) the effects of a backflow transformation of the many-electron wave function. We have also investigated our use of high-symmetry points $(K$ and $M$ ) in the Brillouin zone rather than the true positions of the VBM and CBM at the DFT-HSE06 level of theory.

We find that backflow lowers the DMC quasiparticle $\left(K_{\mathrm{V}} \rightarrow \mathrm{CBM}\right)$ gap of bulk $\mathrm{hBN}$ in the $N_{\mathrm{P}}=9$ supercell by $0.17(5) \mathrm{eV}$. By considering the exact VBM and CBM positions, we find a further energy lowering of $0.02(6) \mathrm{eV}$, which is not statistically significant. Further, we have also considered explicit reoptimization of backflow functions in anionic and cationic states for the VBM $\rightarrow$ CBM quasiparticle gap. This has recently been shown to lead to significant further lowering of SJB-DMC quasiparticle energy gaps [40]; however, in this case we find that reoptimization of the backflow functions by minimizing the VMC energy raises the SJB-DMC gap by $0.08(3) \mathrm{eV}$ as is also found in the monolayer. Near degeneracy of the bands at the $M$ point is a possible cause of both the unusual behavior of the DMC energy in the presence of

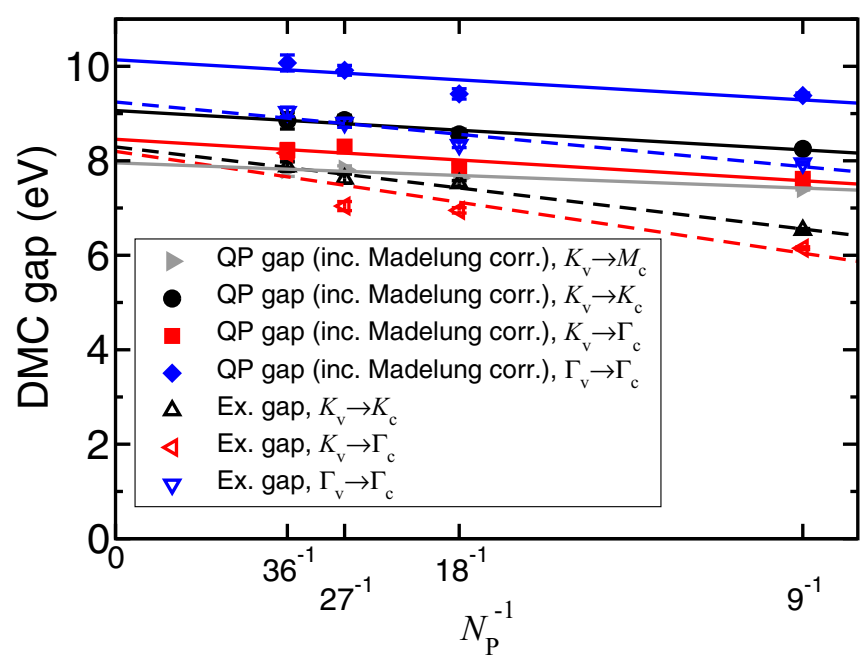

FIG. 9. SJ-DMC quasiparticle gaps $\Delta_{\mathrm{qp}}$ and excitonic gaps $\Delta_{\mathrm{ex}}$ of bulk hBN against $1 / N_{\mathrm{P}}$, where $N_{\mathrm{P}}$ is the number of primitive cells in the supercell. The quasiparticle gaps include the Madelung correction given in Eq. (4). The statistical error bars show the random error in the SJ-DMC gap in a particular supercell; the noise due to quasirandom finite-size effects clearly exceeds the noise due to the Monte Carlo calculation. 
backflow and the overestimate of the gap. Near degeneracy can lead to multireference character and hence significant fixed-node errors with a single-determinant wave function.

\section{CONCLUSIONS}

We have performed DFT, $G W$, and SJ-DMC calculations to determine the electronic structure of free-standing monolayer and bulk hBN. Systematic finite-size errors in the SJ-DMC quasiparticle gaps fall off as the reciprocal of the linear size of the simulation supercell, but can be corrected by subtracting an appropriately screened Madelung constant from the gap. The remaining finite-size effects are dominated by quasirandom oscillations as a function of system size, arising from the fact that long-range oscillations in the pair-correlation function are forced to be commensurate with the supercell. We find the SJ-DMC quasiparticle gap for the monolayer to be indirect $\left(K_{\mathrm{v}} \rightarrow \Gamma_{\mathrm{c}}\right)$ and of magnitude $8.8(3) \mathrm{eV}$, which is larger than the gap predicted by the $G_{0} W_{0}, G W_{0}$, and $G W$ methods. Our bulk SJ-DMC quasiparticle gaps are also systematically larger than those predicted by $G W$ calculations [31]. Using DFT, we also find a sizable vibrational correction to the monolayer band gap of $-0.73 \mathrm{eV}$ at $300 \mathrm{~K}$, and a vibrational correction of $-0.40 \mathrm{eV}$ to the bulk band gap at $300 \mathrm{~K}$.

SJ-DMC shows that hBN exhibits large exciton binding energies of 1.9(4) and 1.8(4) eV for the indirect $\left(K_{\mathrm{v}} \rightarrow \Gamma_{\mathrm{c}}\right)$ and direct $\left(K_{\mathrm{v}} \rightarrow K_{\mathrm{c}}\right)$ excitons in the monolayer. The latter binding energy is similar to the value predicted by our $G W_{0^{-}}$ BSE calculation for the direct exciton and compares well to previous $G W$-BSE calculations [31-33], as well as the exciton binding energy obtained within the effective-mass approximation with the Keldysh interaction between charge carriers [75]. The predicted quasiparticle gaps of $\mathrm{hBN}$ increase significantly as one goes from DFT with local functionals, to DFT with hybrid functionals, to $G_{0} W_{0}$, to $G W_{0}$, to $G W$, to SJ-DMC.

Comparing SJ-DMC gaps with experimental results for bulk hBN shows that the SJ-DMC gaps are significantly too high, even when DFT-calculated vibrational renormalizations are included; the overestimate is around $1.5 \mathrm{eV}$. Several sources of error on a $0.1-0.3 \mathrm{eV}$ energy scale have been identified: uncertainties due to pseudopotentials, residual finite-size errors after extrapolation of the noisy data to infinite system size, and the need for a more complete treatment of dynamical correlation effects through the use of backflow wave functions. In addition, there are unquantified fixed-node errors arising from the use of a single-determinant wave function. Although we investigated very small multideterminant wave functions for the monolayer, it is possible that there could be significant uncanceled fixed-node errors due to multireference character in some of the excited-state wave functions. The mismatch between the minima of the VMC and DMC energies with respect to backflow functions gives some hint that this might be the case. A further possible cause of the disagreement with experiment is the underestimate of the vibrational renormalization of the gap. Several materials exhibit vibrational corrections to the band gap that are up to $50 \%$ (although typically only $10 \%-20 \%$ ) larger when calculated using $G W$ theory or hybrid functionals rather than a semilocal DFT functional $[89,90]$. In the case of $h B N$, vibrational renormalizations of the band gap could therefore be as large as $-1 \mathrm{eV}$ for the monolayer at $300 \mathrm{~K}$ and $-0.5 \mathrm{eV}$ for the bulk at $300 \mathrm{~K}$.

Static-nucleus self-consistent $G W$ calculations agree remarkably well with the experimental quasiparticle gap of bulk hBN, but taking into account vibrational effects we find that the $G W$ quasiparticle gap is underestimated by about $0.4 \mathrm{eV}$. When vibrational effects are included, single-shot $G_{0} W_{0}$ methods underestimate the experimental gap by about $1 \mathrm{eV}$. Determining the electronic structure of hBN from first principles with quantitative accuracy remains a challenging problem.

\section{ACKNOWLEDGMENTS}

We acknowledge financial support from the U. K. Engineering and Physical Sciences Research Council (EPSRC) through a Science and Innovation Award, the E. U. through the grant Concept Graphene, the Royal Society, and Lancaster University through the Early Career Small Grant Scheme. R.J.H. is fully funded by the Graphene NOWNANO CDT (Grant No. EP/L01548X/1). B.M. acknowledges support from the Winton Programme for the Physics of Sustainability, and from Robinson College, Cambridge, and the Cambridge Philosophical Society for a Henslow Research Fellowship. Computational resources were provided by Lancaster University's High-End Computing facility. V.Z. acknowledges the European Graphene Flagship Project and the ARCHER National UK Supercomputer RAP Project e547. This work made use of the facilities of N8 HPC provided and funded by the N8 consortium and EPSRC (Grant No. EP/K000225/1). We acknowledge useful discussions with V. I. Fal'ko, W. M. C. Foulkes, and S. Murphy.
[1] K. S. Novoselov, A. K. Geim, S. V. Morozov, D. Jiang, Y. Zhang, S. V. Dubonos, I. V. Grigorieva, and A. A. Firsov, Science 306, 666 (2004).

[2] A. K. Geim and K. S. Novoselov, Nat. Mater. 6, 183 (2007).

[3] Q. H. Wang, K. Kalantar-Zadeh, A. Kis, J. N. Coleman, and M. S. Strano, Nat. Nanotechnol. 7, 699 (2012).

[4] K. F. Mak, C. Lee, J. Hone, J. Shan, and T. F. Heinz, Phys. Rev. Lett. 105, 136805 (2010).
[5] A. Splendiani, L. Sun, Y. Zhang, T. Li, J. Kim, Ch.-Y. Chim, G. Galli, and F. Wang, Nano Lett. 10, 1271 (2010).

[6] L. Li, Y. Yu, G. J. Ye, Q. Ge, X. Ou, H. Wu, D. Feng, X. H. Chen, and Y. Zhang, Nat. Nanotechnol. 9, 372 (2014).

[7] S. P. Koenig, R. A. Doganov, H. Schmidt, A. H. Castro Neto, and B. Özyilmaz, Appl. Phys. Lett. 104, 103106 (2014).

[8] H. Liu, A. T. Neal, Z. Zhu, Z. Luo, X. Xu, D. Tománek, and P. D. Ye, ACS Nano 8, 4033 (2014). 
[9] A. K. Geim and I. V. Grigorieva, Nature (London) 499, 419 (2013).

[10] C. R. Dean, A. F. Young, I. Meric, C. Lee, L. Wang, S. Sorgenfrei, K. Watanabe, T. Taniguchi, P. Kim, K. L. Shepard, and J. Hone, Nat. Nanotechnol. 5, 722 (2010).

[11] J. M. Xue, J. Sanchez-Yamagishi, D. Bulmash, P. Jacquod, A. Deshpande, K. Watanabe, T. Taniguchi, P. Jarillo-Herrero, and B. J. Leroy, Nat. Mater. 10, 282 (2011).

[12] M. S. Bresnehan, M. J. Hollander, M. Wetherington, M. LaBella, K. A. Trumbull, R. Cavalero, D. W. Snyder, and J. A. Robinson, ACS Nano 6, 5234 (2012).

[13] K. H. Lee, H. J. Shin, J. Lee, I. Y. Lee, G. H. Kim, J. Y. Choi, and S. W. Kim, Nano Lett. 12, 714 (2012).

[14] Z. Liu, L. L. Ma, G. Shi, W. Zhou, Y. J. Gong, S. D. Lei, X. B. Yang, J. N. Zhang, J. J. Yu, K. P. Hackenberg, A. Babakhani, J. C. Idrobo, R. Vajtai, J. Lou, and P. M. Ajayan, Nat. Nanotechnol. 8, 119 (2013).

[15] L. Britnell, R. V. Gorbachev, R. Jalil, B. D. Belle, F. Schedin, A. Mishchenko, T. Georgiou, M. I. Katsnelson, L. Eaves, S. V. Morozov, N. M. R. Peres, J. Leist, A. K. Geim, K. S. Novoselov, and L. A. Ponomarenko, Science 335, 947 (2012).

[16] L. Britnell, R. V. Gorbachev, A. K. Geim, L. A. Ponomarenko, A. Mishchenko, M. T. Greenaway, T. M. Fromhold, K. S. Novoselov, and L. Eaves, Nat. Commun. 4, 1794 (2013).

[17] G. Shi, Y. Hanlumyuang, Z. Liu, Y. Gong, W. Gao, B. Li, J. Kono, J. Lou, R. Vajtai, P. Sharma, and P. M. Ajayan, Nano Lett. 14, 1739 (2014).

[18] M. Yankowitz, J. Xue, D. Cormode, J. D. Sanchez-Yamagishi, K. Watanabe, T. Taniguchi, P. Jarillo-Herrero, P. Jacquod, and B. J. LeRoy, Nat. Phys. 8, 382 (2012).

[19] D. Hofstadter, Phys. Rev. B 14, 2239 (1976).

[20] L. A. Ponomarenko, R. V. Gorbachev, G. L. Yu, D. C. Elias, R. Jalil, A. A. Patel, A. Mishchenko, A. S. Mayorov, C. R. Woods, J. R. Wallbank, M. Mucha-Kruczynski, B. A. Piot, M. Potemski, I. V. Grigorieva, K. S. Novoselov, F. Guinea, V. I. Fal'ko, and A. K. Geim, Nature (London) 497, 594 (2013).

[21] C. R. Dean, L. Wang, P. Maher, C. Forsythe, F. Ghahari, Y. Gao, J. Katoch, M. Ishigami, P. Moon, M. Koshino, T. Taniguchi, K. Watanabe, K. L. Shepard, J. Hone, and P. Kim, Nature (London) 497, 598 (2013).

[22] X. Blase, A. Rubio, S. G. Louie, and M. L. Cohen, Phys. Rev. B 51, 6868 (1995).

[23] D. M. Hoffman, G. L. Doll, and P. C. Eklund, Phys. Rev. B 30, 6051 (1984).

[24] L. G. Carpenter and P. J. Kirby, J. Phys. D: Appl. Phys. 15, 1143 (1982).

[25] K. Watanabe, T. Taniguchi, and H. Kanda, Nat. Mater. 3, 404 (2004).

[26] Y. Shi, C. Hamsen, X. Jia, K. K. Kim, A. Reina, M. Hofmann, A. L. Hsu, K. Zhang, H. Li, Z.-Y. Juang, M. S. Dresselhaus, L.-J. Li, and J. Kong, Nano Lett. 10, 4134 (2010).

[27] G. Cassabois, P. Valvin, and B. Gil, Nat. Photonics 10, 262 (2016).

[28] E. Cannuccia, B. Monserrat, and C. Attaccalite, Phys. Rev. B 99, 081109(R) (2019).

[29] F. Paleari, H. P. C. Miranda, A. Molina-Sánchez, and L. Wirtz, Phys. Rev. Lett. 122, 187401 (2019).

[30] G. Cappellini, V. Fiorentini, K. Tenelsen, and F. Bechstedt, in Gallium Nitride and Related Materials, edited by R. D. Dupuis, J. A. Edmond, F. A. Ponce, and S. Nakamura, MRS Symposia
Proceedings No. 395 (Materials Research Society, Pittsburgh, 1996).

[31] B. Arnaud, S. Lebègue, P. Rabiller, and M. Alouani, Phys. Rev. Lett. 96, 026402 (2006).

[32] L. Wirtz, A. Marini, and A. Rubio, Phys. Rev. Lett. 96, 126104 (2006).

[33] B. Cunningham, M. Grüning, P. Azarhoosh, D. Pashov, and M. van Schilfgaarde, Phys. Rev. Mater. 2, 034603 (2018).

[34] C. Elias, P. Valvin, T. Pelini, A. Summerfield, C. J. Mellor, T. S. Cheng, L. Eaves, C. T. Foxon, P. H. Beton, S. V. Novikov, B. Gil, and G. Cassabois, Nat. Commun. 10, 2639 (2019).

[35] L. Hedin, Phys. Rev. 139, A796 (1965).

[36] D. M. Ceperley and B. J. Alder, Phys. Rev. Lett. 45, 566 (1980).

[37] W. M. C. Foulkes, L. Mitas, R. J. Needs, and G. Rajagopal, Rev. Mod. Phys. 73, 33 (2001).

[38] J. B. Anderson, J. Chem. Phys. 65, 4121 (1976).

[39] R. J. Needs, M. D. Towler, N. D. Drummond, and P. López Ríos, J. Phys.: Condens. Matter 22, 023201 (2010).

[40] R. J. Hunt, M. Szyniszewski, G. I. Prayogo, R. Maezono, and N. D. Drummond, Phys. Rev. B 98, 075122 (2018).

[41] T. Frank, R. Derian, K. Tokár, L. Mitas, J. Fabian, and I. Štich, Phys. Rev. X 9, 011018 (2019).

[42] J. P. Perdew, K. Burke, and M. Ernzerhof, Phys. Rev. Lett. 77, 3865 (1996).

[43] J. Heyd, G. E. Scuseria, and M. Ernzerhof, J. Chem. Phys. 118, 8207 (2003).

[44] A. V. Krukau, O. A. Vydrov, A. F. Izmaylov, and G. E. Scuseria, J. Chem. Phys. 125, 224106 (2006).

[45] S. J. Clark, M. D. Segall, C. J. Pickard, P. J. Hasnip, M. I. J. Probert, K. Refson, and M. C. Payne, Z. Kristallogr. 220, 567 (2005).

[46] G. Kresse and J. Furthmüller, Phys. Rev. B 54, 11169 (1996).

[47] K. Refson, P. R. Tulip, and S. J. Clark, Phys. Rev. B 73, 155114 (2006).

[48] J. R. Trail and R. J. Needs, J. Chem. Phys. 122, 014112 (2005).

[49] J. R. Trail and R. J. Needs, J. Chem. Phys. 122, 174109 (2005).

[50] M. Shishkin and G. Kresse, Phys. Rev. B 74, 035101 (2006).

[51] J. Deslippe, G. Samsonidze, D. A. Strubbe, M. Jain, M. L. Cohen, and S. G. Louie, Comput. Phys. Commun. 183, 1269 (2012).

[52] L. Mitas and R. M. Martin, Phys. Rev. Lett. 72, 2438 (1994).

[53] A. J. Williamson, R. Q. Hood, R. J. Needs, and G. Rajagopal, Phys. Rev. B 57, 12140 (1998).

[54] M. D. Towler, R. Q. Hood, and R. J. Needs, Phys. Rev. B 62, 2330 (2000).

[55] W. M. C. Foulkes, R. Q. Hood, and R. J. Needs, Phys. Rev. B 60, 4558 (1999).

[56] D. Alfè and M. J. Gillan, Phys. Rev. B 70, 161101(R) (2004).

[57] N. D. Drummond, M. D. Towler, and R. J. Needs, Phys. Rev. B 70, 235119 (2004).

[58] C. J. Umrigar, K. G. Wilson, and J. W. Wilkins, Phys. Rev. Lett. 60, 1719 (1988).

[59] N. D. Drummond and R. J. Needs, Phys. Rev. B 72, 085124 (2005).

[60] Y. Kwon, D. M. Ceperley, and R. M. Martin, Phys. Rev. B 48, 12037 (1993).

[61] P. López Ríos, A. Ma, N. D. Drummond, M. D. Towler, and R. J. Needs, Phys. Rev. E 74, 066701 (2006).

[62] C. J. Umrigar, J. Toulouse, C. Filippi, S. Sorella, and R. G. Hennig, Phys. Rev. Lett. 98, 110201 (2007). 
[63] L. Mitáš, E. L. Shirley, and D. M. Ceperley, J. Chem. Phys. 95, 3467 (1991).

[64] E. L. Shirley and R. M. Martin, Phys. Rev. B 47, 15413 (1993).

[65] F. Marsusi, J. Sabbaghzadeh, and N. D. Drummond, Phys. Rev. B 84, 245315 (2011).

[66] J. H. Lloyd-Williams and B. Monserrat, Phys. Rev. B. 92, 184301 (2015).

[67] G. Rajagopal, R. J. Needs, S. Kenny, W. M. C. Foulkes, and A. James, Phys. Rev. Lett. 73, 1959 (1994).

[68] G. Rajagopal, R. J. Needs, A. James, S. D. Kenny, and W. M. C. Foulkes, Phys. Rev. B 51, 10591 (1995).

[69] E. Madelung, Phys. Zs. XIX, 524 (1918).

[70] N. D. M. Hine, K. Frensch, W. M. C. Foulkes, and M. W. Finnis, Phys. Rev. B 79, 024112 (2009).

[71] L. V. Keldysh, Pis'ma Zh. Eksp. Teor. Fiz. 29, 716 (1979) [J. Exp. Theor. Phys. 29, 658 (1979)].

[72] B. Ganchev, N. Drummond, I. Aleiner, and V. Fal'ko, Phys. Rev. Lett. 114, 107401 (2015).

[73] R. W. Lynch and H. G. Drickamer, J. Chem. Phys. 44, 181 (1966). Note that the $a$ and $c$ lattice parameters are interchanged in Table II.

[74] S. T. Murphy and N. D. M. Hine, Phys. Rev. B 87, 094111 (2013).

[75] E. Mostaani, M. Szyniszewski, C. H. Price, R. Maezono, M. Danovich, R. J. Hunt, N. D. Drummond, and V. I. Fal'ko, Phys. Rev. B 96, 075431 (2017).

[76] B. Monserrat, J. Phys.: Condens. Matter 30, 083001 (2018).

[77] B. Monserrat, Phys. Rev. B 93, 014302 (2016).
[78] B. Monserrat, E. A. Engel, and R. J. Needs, Phys. Rev. B 92, 140302(R) (2015).

[79] W. A. Saidi, S. Poncé, and B. Monserrat, J. Phys. Chem. Lett. 7, 5247 (2016).

[80] A. Tkatchenko and M. Scheffler, Phys. Rev. Lett. 102, 073005 (2009).

[81] R. M. Ribeiro and N. M. R. Peres, Phys. Rev. B 83, 235312 (2011).

[82] R. S. Mulliken, J. Chem. Phys. 23, 1833 (1955).

[83] F. L. Hirshfeld, Theor. Chim. Acta 44, 129 (1977).

[84] V. Zólyomi, N. D. Drummond, and V. I. Fal'ko, Phys. Rev. B 89, 205416 (2014).

[85] L. Wirtz, A. Rubio, R. A. de la Concha, and A. Loiseau, Phys. Rev. B 68, 045425 (2003).

[86] L. Wirtz, M. Lazzeri, F. Mauri, and A. Rubio, Phys. Rev. B 71, 241402(R) (2005).

[87] F. Paleari, T. Galvani, H. Amara, F. Ducastelle, A. MolinaSánchez, and L. Wirtz, 2D Mater. 5, 045017 (2018).

[88] F. Giustino, S. G. Louie, and M. L. Cohen, Phys. Rev. Lett. 105, 265501 (2010).

[89] G. Antonius, S. Poncé, P. Boulanger, M. Côté, and X. Gonze, Phys. Rev. Lett. 112, 215501 (2014).

[90] B. Monserrat, Phys. Rev. B 93, 100301(R) (2016).

[91] M. Casula, Phys. Rev. B 74, 161102(R) (2006).

[92] M. Casula, S. Moroni, S. Sorella, and C. Filippi, J. Chem. Phys. 132, 154113 (2010).

[93] N. D. Drummond, J. R. Trail, and R. J. Needs, Phys. Rev. B 94, 165170 (2016). 